\title{
Uniqueness, Spatial Mixing, and Approximation for Ferromagnetic 2-Spin Systems
}

\author{
Heng Guo*1 and Pinyan $\mathrm{Lu}^{2}$
}

1 School of Mathematical Sciences, Queen Mary University of London, U.K. h.guo@qmul.ac.uk

2 The Institute for Theoretical Computer Science, School of Information Management and Engineering, Shanghai University of Finance and Economics, China

lu.pinyan@mail.shufe.edu.cn

\begin{abstract}
For anti-ferromagnetic 2-spin systems, a beautiful connection has been established, namely that the following three notions align perfectly: the uniqueness of Gibbs measures in infinite regular trees, the decay of correlations (also known as spatial mixing), and the approximability of the partition function. The uniqueness condition implies spatial mixing, and an FPTAS for the partition function exists based on spatial mixing. On the other hand, non-uniqueness implies some long range correlation, based on which NP-hardness reductions are built.

These connections for ferromagnetic 2-spin systems are much less clear, despite their similarities to anti-ferromagnetic systems. The celebrated Jerrum-Sinclair Markov chain [8] works even if spatial mixing fails. Also, for a fixed degree the uniqueness condition is non-monotone with respect to the external field, which seems to have no meaningful interpretation in terms of computational complexity. However, it is still intriguing whether there are some relationship underneath the apparent disparities among them.

We provide some answers to this question. Let $\beta, \gamma$ be the $(0,0)$ and $(1,1)$ edge interactions respectively $(\beta \gamma>1)$, and $\lambda$ the external field for spin "0". For graphs with degree bound $\Delta \leq \Delta_{c}+1$ where $\Delta_{c}=\frac{\sqrt{\beta \gamma}+1}{\sqrt{\beta \gamma}-1}$, regardless of the field (even inconsistent fields are allowed) correlation decay always holds and FPTAS exists. If all fields satisfy $\lambda<\lambda_{c}$ (assuming $\beta \leq \gamma$ ), where $\lambda_{c}=(\gamma / \beta)^{\frac{\Delta_{c}+1}{2}}$, then a weaker version of spatial mixing holds in all trees. Moreover, if $\beta \leq 1$, then $\lambda<\lambda_{c}$ is sufficient to guarantee strong spatial mixing and FPTAS. This improves the best previous algorithm, a Markov chain based FPRAS for $\lambda \leq \gamma / \beta$ [13].

The bound $\lambda_{c}$ is almost optimal and can be viewed as a variant of the uniqueness condition with the degree $d$ relaxed to be a real number instead of an integer. When $\beta \leq 1$, uniqueness holds in all infinite regular trees, if and only if $\lambda \leq \lambda_{c}^{i n t}$, where $\lambda_{c}^{i n t}=(\gamma / \beta)^{\frac{\left[\Delta_{c}\right]+1}{2}}$. If we allow fields $\lambda>\lambda_{c}^{i n t^{\prime}}$, where $\lambda_{c}^{i n t^{\prime}}=(\gamma / \beta)^{\frac{\left\lfloor\Delta_{c}\right\rfloor+2}{2}}$, then approximating the partition function is \#BIS-hard.

Interestingly, unless $\Delta_{c}$ is an integer, neither $\lambda_{c}$ nor $\lambda_{c}^{i n t}$ is the tight bound in each own respect. We provide examples where correlation decay continues to hold in a small interval beyond $\lambda_{c}$, and irregular trees in which spatial mixing fails for some $\lambda<\lambda_{c}^{i n t}$.
\end{abstract}

1998 ACM Subject Classification F.2.2 Nonnumerical Algorithms and Problems, G.2.1 Combinatorics

Keywords and phrases Approximate counting, Ising model, Spin systems, Correlation decay

Digital Object Identifier 10.4230/LIPIcs.APPROX-RANDOM.2016.31

* HG is supported by EPSRC Standard Research Grant EP/N004221/1. 


\section{Introduction}

Spin systems model nearest neighbor interactions. In this paper we study 2-state spin systems. An instance is a graph $G=(V, E)$, and a configuration $\sigma$ assigns one of the two spins " 0 " and " 1 " to each vertex; that is, $\sigma$ is one of the $2^{|V|}$ possible assignments $\sigma: V \rightarrow\{0,1\}$. The local interaction along an edge is specified by a matrix $\mathbf{A}=\left[\begin{array}{ll}A_{0,0} & A_{0,1} \\ A_{1,0} & A_{1,1}\end{array}\right]$, where $A_{i, j}$ is the (non-negative) local weight when the two endpoints are assigned $i$ and $j$ respectively. We study symmetric edge interactions, that is, $A_{0,1}=A_{1,0}$. Normalize $\mathbf{A}$ so that $\mathbf{A}=\left[\begin{array}{ll}\beta & 1 \\ 1 & \gamma\end{array}\right]$. Moreover, we also consider the external field, specified by a mapping $\pi: V \rightarrow \mathbb{R}^{+}$. When a vertex is assigned " 0 ", we give it a weight $\pi(v)$. For a particular configuration $\sigma$, its weight $w(\sigma)$ is a product over all edge interactions and vertex weights, that is

$$
w(\sigma)=\beta^{m_{0}(\sigma)} \gamma^{m_{1}(\sigma)} \prod_{v \mid \sigma(v)=0} \pi(v),
$$

where $m_{0}(\sigma)$ is the number of $(0,0)$ edges under the configuration $\sigma$ and $m_{1}(\sigma)$ is the number of $(1,1)$ edges. An important special case is the Ising model, where $\beta=\gamma$. The Gibbs measure is a natural distribution in which each configuration $\sigma$ is drawn with probability proportional to its weight, that is, $\operatorname{Pr}_{G ; \beta, \gamma, \pi}(\sigma) \sim w(\sigma)$. The normalizing factor of the Gibbs measure is called the partition function, defined by $Z_{\beta, \gamma, \pi}(G)=\sum_{\sigma: V \rightarrow\{0,1\}} w(\sigma)$. The partition function encodes rich information regarding the macroscopic behavior of the spin system. We will be interested in the computational complexity of approximating $Z_{\beta, \gamma, \pi}(G)$. We also simply write $Z_{\beta, \gamma, \lambda}(G)$ when the field is uniform, that is, $\pi(v)=\lambda$ for all $v \in V$. A system with uniform fields is specified by the three parameters $(\beta, \gamma, \lambda)$.

Spin systems not only are interesting in statistical physics, but also find applications in computer science, under the name of Markov random fields. In fact, a 2-state spin system is equivalent to a binary Markov random field, and computing the partition function is central to statistical inference. According to their physical and computational properties, spin systems can be classified into two families: ferromagnetic systems where the edge interaction is attractive $(\beta \gamma>1)$, and anti-ferromagnetic systems where it is repulsive $(\beta \gamma<1)$.

Recently, beautiful connections have been established regarding three different aspects of anti-ferromagnetic 2-spin systems. The uniqueness of Gibbs measures in infinite regular trees $^{1}$ of degrees up to $\Delta$ implies correlation decay ${ }^{2}$ in all graphs of maximum degree $\Delta$, and therefore the existence of fully polynomial-time approximation scheme (FPTAS) for the partition function $[19,11,17,12]$. On the other hand, if the tree uniqueness fails, then long range correlation appears and the partition function has no fully polynomial-time randomized approximation scheme (FPRAS) unless $\mathrm{NP}=\mathrm{RP}[18,4]$. It suggests that the mathematical property of tree uniqueness, the physical property of spatial mixing, and the computational complexity of approximating the partition function, line up perfectly in anti-ferromagnetic 2-spin systems.

For ferromagnetic systems, the picture is much less clear. In a seminal paper [8], Jerrum and Sinclair gave an FPRAS for the ferromagnetic Ising model $\beta=\gamma>1$ with any consistent external field $\lambda$ for general graphs without degree bounds. Thus, there is no computational complexity transition of approximating these models, whereas uniqueness and spatial mixing do exhibit phase transition. This is in sharp contrast to anti-ferromagnetic Ising models

1 This property is called "tree uniqueness" or "uniqueness" for short. See Sections 2.2 and 6.1 for details.

2 That is, the correlation of any two vertices decay exponentially in distance. It is also called "spatial mixing". 
$\beta=\gamma<1$, where computational and phase transitions align perfectly. It is not clear at all whether spatial mixing or correlation decay plays any role in the computational complexity.

For more general ferromagnetic 2-spin systems with external fields, the threshold for approximating the partition function is still open. On the complexity side, Goldberg and Jerrum showed that any ferromagnetic 2-spin system is no harder than counting independent sets in bipartite graphs (\#BIS) [6], which is conjectured to have no FPRAS [3] (the approximation complexity of \#BIS is still open). Based on an earlier result [1], Liu, Lu and Zhang showed that approximating the partition function is \#BIS-hard if we allow external fields beyond $(\gamma / \beta)^{\frac{\left\lfloor\Delta_{c}\right\rfloor+2}{2}}$ where $\Delta_{c}=\frac{\sqrt{\beta \gamma}+1}{\sqrt{\beta \gamma}-1}[13] .^{3}$

On the algorithmic side, by reducing to the Ising model, an MCMC based FPRAS is known for the range of $\lambda \leq \sqrt{\gamma / \beta}$ [7], which has been recently improved to $\lambda \leq \gamma / \beta[13]$. On the other hand, if we apply the correlation decay algorithmic framework to various pairs of parameters $(\beta, \gamma)$, it is not hard to get bounds better than $\gamma / \beta$. However, such success for individual problems does not seem to share meaningful inner connections. In particular, it is not clear how far one can push this method, and to the best of our knowledge, no threshold has even been conjectured.

\subsection{Our Contribution}

In this paper, we identify a new threshold that almost tightly maps out the boundary of the correlation decay regime, that is, $\lambda_{c}=(\gamma / \beta)^{\frac{\Delta_{c}+1}{2}}=(\gamma / \beta)^{\frac{\sqrt{\beta \gamma}}{\sqrt{\beta \gamma}-1}}$. We show that for any $\lambda<\lambda_{c}$ a variant of spatial mixing holds (Theorem 1) for arbitrary trees. An interesting feature of our work is that we do not restrict the degree or the shape of the tree. This is almost tight since it does not hold if $\lambda>(\gamma / \beta)^{\frac{\left[\Delta_{c}\right\rceil+1}{2}}$. This spatial mixing is weaker than what an algorithm usually requires, but in the regime of $\beta \leq 1$ it implies (and therefore is equivalent to) strong spatial mixing. As an algorithmic consequence, we have FPTAS for all $\beta \leq 1<\gamma, \beta \gamma>1$, and $\lambda<\lambda_{c}$ (Theorem 2). Recall that if we allow $\lambda$ beyond $(\gamma / \beta)^{\frac{\left\lfloor\Delta_{c}\right\rfloor+2}{2}}$, then the problem is \#BIS-hard [13]. Hence only an integral gap remains for the $\beta \leq 1<\gamma$ case.

Formally, let $p_{v}$ be the marginal probability of $v$ (being assigned "0").

Theorem 1. Let $(\beta, \gamma, \lambda)$ be a set of parameters of the system such that $\beta \gamma>1, \beta \leq \gamma$, and $\lambda<\lambda_{c}$. Let $T_{v}$ and $T_{v^{\prime}}^{\prime}$ be two trees with roots $v$ and $v^{\prime}$ respectively. If the two trees have the same structure in the first $\ell$ levels, then $\left|p_{v}-p_{v^{\prime}}\right| \leq O(\exp (-\ell))$.

In other words, if we simply truncate a tree at depth $\ell$, the marginal probability of its root will change by only at most $O(\exp (-\ell))$. Surprisingly, if we replace $\lambda_{c}$ by its integral counterpart, then this implication no longer holds and there is a counterexample (see Section 5). More precisely, it is no longer true that the uniqueness in infinite regular trees implies correlation decay in graphs or even trees, since our counterexample is an irregular tree. We note that this is in sharp contrast to anti-ferromagnetic systems, where (integral) uniqueness implies correlation decay.

From the computational complexity point of view, we would like to get FPTAS for the partition function, which requires a condition called strong spatial mixing (SSM). It is stronger than the spatial mixing established in Theorem 1 by imposing arbitrary partial configurations. We are able to prove SSM with $\lambda<\lambda_{c}$ for the range of $\beta \leq 1$. Indeed, if

${ }^{3}$ Here and below we assume $\beta \leq \gamma$ due to symmetry. 
$\beta \leq 1$, then the two versions of spatial mixing are equivalent. Let $I$ be an interval of the form $\left[\lambda_{1}, \lambda_{2}\right]$ or $\left(\lambda_{1}, \lambda_{2}\right]$. We consider the following problem.

Name: \#2Spin $(\beta, \gamma, I)$

Instance: A graph $G=(V, E)$ and a mapping $\pi: V \rightarrow \mathbb{R}^{+}$, such that $\pi(v) \in I$ for any $v \in V$.

Output: $Z_{\beta, \gamma, \pi}(G)$.

Then we have the following theorem.

- Theorem 2. Let $(\beta, \gamma, \lambda)$ be a set of parameters of the system such that $\beta \gamma>1, \beta \leq 1$ and $\lambda<\lambda_{c}$. Then \#2SPIN $(\beta, \gamma,(0, \lambda])$ has an FPTAS.

Therefore, we get an almost tight dichotomy for ferromagnetic 2 -spin systems when $\beta \leq 1$, since \#2SPIN $(\beta, \gamma,(0, \lambda])$ is \#BIS-hard, if $\lambda$ is larger than the integral counterpart of $\lambda_{c}[13]$ (see also Proposition 22).

The reason behind $\lambda_{c}$ is a nice interplay among uniqueness, spatial mixing, and approximability. We start with some purely mathematical observations on the symmetric tree recursion $f_{d}(x)=\lambda\left(\frac{\beta x+1}{x+\gamma}\right)^{d}$, an increasing function in $x$. Relax the range of $d$ in $f_{d}(x)$ to be real numbers. Then $\Delta_{c}$ is the critical (possibly fractional) degree and $\lambda_{c}$ is the corresponding critical external field for the recursion to have a unique fixed point. This set of critical parameters enjoys some very nice mathematical properties. For $d=\Delta_{c}$ and $\lambda=\lambda_{c}$, the function $f_{d}(x)$ has a unique fixed point $\widehat{x}=\sqrt{\gamma / \beta}$ and $f_{d}^{\prime}(\widehat{x})=1$. Moreover, it also satisfies that $f_{d}^{\prime \prime}(\widehat{x})=0$, which is a necessary condition for the contraction of the tree recursion (easily derived using the heuristic of finding potential functions described in [12]). All these nice mathematical properties prove to be useful in our later analysis. For degrees other than $\Delta_{c}$, their critical external fields are much less convenient - the function $f_{d}(x)$ has two fixed points: one is crossing and the other is tangent. Moreover, $f_{d}^{\prime \prime}(\hat{x})=0$ does not necessarily hold.

The proof of Theorem 1 uses the potential method to analyze decay of correlation, which is now streamlined (see e.g. [12]). The main difficulty is to find a good potential function. In other words, we want to solve a variational problem minimizing the maximum of the decay rate function. The main novelty in our solution is that we restrict variables to the range of $\left(0, \frac{\lambda}{1+\lambda}\right]$ and our potential function is well-defined only in this range. This is in fact necessary, as otherwise the statement does not hold, and is valid for the setting of Theorem 1. Also note that with our choice, the proof is relatively clean and significantly simpler than similar proofs in other settings. In particular, we do not need the "symmetrization" argument (see e.g. $[12,16])$. We also use a trick of truncating the potential to deal with unbounded degrees (see Eq. (5)).

For the range of $\beta>1$, SSM does not hold even if $\lambda<\lambda_{c}$. However, we conjecture that Theorem 2 can be extended to the $\beta>1$ range as well, mainly due to Theorem 1 , which does not require $\beta \leq 1$. Moreover, we show that even if $\beta>1$, the marginal probability in any instance is within the range of $\left(0, \frac{\lambda}{1+\lambda}\right]$ given $\lambda<\lambda_{c}$ (see Proposition 20). This seems to imply that the main reason why our algorithm fails is due to pinnings (forcing a vertex to be "0" or "1") in the self-avoiding walk tree construction, whereas in a real instance these pinnings cannot aggregate enough "bad" influence. However, to turn such intuition into an algorithm requires a careful treatment of these pinnings to achieve an FPTAS without SSM. We leave this as an important open question.

At last, we note that neither $\lambda_{c}$ nor its integral counterpart is the exact threshold in each own respect, even if $\beta \leq 1$. Strong spatial mixing continues to hold even if $\lambda>\lambda_{c}$ in a small 
interval. We give a concrete example to illustrate this point in Section 4, Proposition 21. Moreover, as mentioned earlier, an irregular tree exists where the correlation decay threshold is lower than the threshold for all infinite regular trees. This is discussed in Section 5. It is another important open question to figure out the exact threshold between $\lambda_{c}$ and its integral counterpart(s).

\section{Preliminaries}

An instance of a 2-spin system is a graph $G=(V, E)$. A configuration $\sigma: V \rightarrow\{0,1\}$ assigns one of the two spins " 0 " and " 1 " to each vertex. We normalize the edge interaction to be $\left[\begin{array}{ll}\beta & 1 \\ 1 & \gamma\end{array}\right]$, and also consider the external field, specified by a mapping $\pi: V \rightarrow \mathbb{R}^{+}$. When a vertex is assigned " 0 ", we give it a weight $\pi(v)$. All parameters are non-negative. For a particular configuration $\sigma$, its weight $w(\sigma)$ is a product over all edge interactions and vertex weights, that is

$$
w(\sigma)=\beta^{m_{0}(\sigma)} \gamma^{m_{1}(\sigma)} \prod_{v \mid \sigma(v)=1} \pi(v)
$$

where $m_{0}(\sigma)$ is the number of $(0,0)$ edges given by the configuration $\sigma$ and $m_{1}(\sigma)$ is the number of $(1,1)$ edges. An important special case is the Ising model, where $\beta=\gamma$. Notice that in the statistic physics literature, parameters are usually chosen to be the logarithms of our parameters above. Different parameterizations do not affect the complexity of the same system.

We also write $\lambda_{v}:=\pi(v)$. If $\pi$ is a constant function such that $\lambda_{v}=\lambda>0$ for all $v \in V$, we also denote it by $\lambda$. We say $\pi$ has a lower bound (or an upper bound) $\lambda>0$, if $\pi$ satisfies the guarantee that $\lambda_{v} \geq \lambda\left(\right.$ or $\left.\lambda_{v} \leq \lambda\right)$.

The Gibbs measure is a natural distribution in which each configuration $\sigma$ is drawn with probability proportional to its weight, that is, $\operatorname{Pr}_{G ; \beta, \gamma, \pi}(\sigma) \sim w(\sigma)$. The normalizing factor of the Gibbs measure is called the partition function, defined by $Z_{\beta, \gamma, \pi}(G)=\sum_{\sigma: V \rightarrow\{0,1\}} w(\sigma)$. Recall that we are interested in the computational problem $\# 2 \operatorname{SPIN}(\beta, \gamma, I)$, where $I$ is an interval of the form $\left[\lambda_{1}, \lambda_{2}\right]$ or $\left(\lambda_{1}, \lambda_{2}\right]$, for which $Z_{\beta, \gamma, \pi}(G)$ is the output. When input graphs are restricted to have a degree bound $\Delta$, we write $\# \Delta-2 \operatorname{SPIN}(\beta, \gamma, I)$ to denote the problem. When the field is uniform, that is, $\lambda$ is the only element in $I$, we $\operatorname{simply}$ write $\# 2 \operatorname{SPIN}(\beta, \gamma, \lambda)$. Due to [2] and a standard diagonal transformation, for any constant $\lambda>0, \# 2 \operatorname{SPIN}(\beta, \gamma, \lambda)$ is \#P-hard unless $\beta=\gamma=0$ or $\beta \gamma=1$.

\subsection{The Self-Avoiding Walk Tree}

We briefly describe Weitz's algorithm [19]. Our algorithms presented later will follow roughly the same paradigm.

The Gibbs measure defines a marginal distribution of spins for each vertex. Let $p_{v}$ denote the probability of a vertex $v$ being assigned "0". Since the system is self-reducible, $\# 2 \operatorname{SpIN}(\beta, \gamma, \lambda)$ is equivalent to computing $p_{v}$ for any vertex $v$ [9] (for details, see for example Lemma 8).

Let $\sigma_{\Lambda} \in\{0,1\}^{\Lambda}$ be a configuration of $\Lambda \subset V$. We call vertices in $\Lambda$ fixed and other vertices free. We use $p_{v}^{\sigma_{\Lambda}}$ to denote the marginal probability of $v$ being assigned " 0 " conditional on the configuration $\sigma_{\Lambda}$ of $\Lambda$.

Suppose the instance is a tree $T$ with root $v$. Let $R_{T}^{\sigma_{\Lambda}}:=p_{v}^{\sigma_{\Lambda}} /\left(1-p_{v}^{\sigma_{\Lambda}}\right)$ be the ratio between the two probabilities that the root $v$ is 0 and 1, while imposing some condition $\sigma_{\Lambda}$ 
(with the convention that $R_{T}^{\sigma_{\Lambda}}=\infty$ when $p_{v}^{\sigma_{\Lambda}}=1$ ). Suppose that $v$ has $d$ children $v_{i}, \ldots v_{d}$. Let $T_{i}$ be the subtree with root $v_{i}$. Due to the independence of subtrees, it is straightforward to get the following recursion for calculating $R_{T}^{\sigma_{\Lambda}}$ :

$$
R_{T}^{\sigma_{\Lambda}}=F_{d}\left(R_{T_{1}}^{\sigma_{\Lambda}}, \ldots, R_{T_{d}}^{\sigma_{\Lambda}}\right),
$$

where the function $F_{d}\left(x_{1}, \ldots, x_{d}\right)$ is defined as

$$
F_{d}\left(x_{1}, \ldots, x_{d}\right):=\lambda_{v} \prod_{i=1}^{d} \frac{\beta x_{i}+1}{x_{i}+\gamma} .
$$

We allow $x_{i}$ 's to take the value $\infty$ as in that case the function $F_{d}$ is clearly well defined. In general we use capital letters like $F, G, C, \ldots$ to denote multivariate functions, and small letters $f, g, c, \ldots$ to denote their symmetric versions, where all variables take the same value. Here we define $f_{d}(x):=\lambda\left(\frac{\beta x+1}{x+\gamma}\right)^{d}$ to be the symmetric version of $F_{d}(\mathbf{x})$.

Let $G(V, E)$ be a graph. Similarly define $R_{G, v}^{\sigma_{\Lambda}}:=p_{v}^{\sigma_{\Lambda}} /\left(1-p_{v}^{\sigma_{\Lambda}}\right)$. In contrast to the case of trees, there is no easy recursion to calculate $R_{G, v}^{\sigma_{\Lambda}}$ for a general graph $G$. This is because of dependencies introduced by cycles. Weitz [19] reduced computing the marginal distribution of $v$ in a general graph $G$ to that in a tree, called the self-avoiding walk (SAW) tree, denoted by $T_{\mathrm{SAW}}(G, v)$. To be specific, given a graph $G=(V, E)$ and a vertex $v \in V, T_{\mathrm{SAW}}(G, v)$ is a tree with root $v$ that enumerates all self-avoiding walks originating from $v$ in $G$, with additional vertices closing cycles as leaves of the tree. Each vertex in the new vertex set $V_{\mathrm{SAW}}$ of $T_{\mathrm{SAW}}(G, v)$ corresponds to a vertex in $G$, but a vertex in $G$ may be mapped to more than one vertices in $V_{\mathrm{SAW}}$. A boundary condition is imposed on leaves in $V_{\mathrm{SAW}}$ that close cycles. The imposed colors of such leaves depend on whether the cycle is formed from a small vertex to a large vertex or conversely, where the ordering is arbitrarily chosen in $G$. Vertex sets $S \subset \Lambda \subset V$ are mapped to respectively $S_{\mathrm{SAW}} \subset \Lambda_{\mathrm{SAW}} \subset V_{\mathrm{SAW}}$, and any configuration $\sigma_{\Lambda} \in\{0,1\}^{\Lambda}$ is mapped to $\sigma_{\Lambda_{\mathrm{SAW}}} \in\{0,1\}^{\Lambda_{\mathrm{SAW}}}$. With slight abuse of notations we may write $S=S_{\mathrm{SAW}}$ and $\sigma_{\Lambda}=\sigma_{\Lambda_{\mathrm{SAW}}}$ when no ambiguity is caused.

- Proposition 3 (Theorem 3.1 of Weitz [19]). Let $G=(V, E)$ be a graph, $v \in V, \sigma_{\Lambda} \in\{0,1\}^{\Lambda}$ be a configuration on $\Lambda \subset V$, and $S \subset V$. Let $T=T_{\mathrm{SAW}}(G, v)$ be constructed as above. It holds that

$$
R_{G, v}^{\sigma_{\Lambda}}=R_{T}^{\sigma_{\Lambda}} .
$$

Moreover, the maximum degree of $T$ is at most the maximum degree of $G$, $\operatorname{dist}_{G}(v, S)=$ $\operatorname{dist}_{T}\left(v, S_{S A W}\right)$, and any neighborhood of $v$ in $T$ can be constructed in time proportional to the size of the neighborhood.

The SAW tree construction does not solve a \#P-hard problem, since $T_{\mathrm{SAW}}(G, v)$ is potentially exponentially large in size of $G$. For a polynomial time approximation algorithm, we may run the tree recursion within some polynomial size, or equivalently a logarithmic depth. At the boundary where we stop, we plug in some arbitrary values. The question is then how large is the error due to our random guess. To guarantee the performance of the algorithm, we need the following notion of strong spatial mixing.

- Definition 4. A spin system on a family $\mathcal{G}$ of graphs is said to exhibit strong spatial mixing (SSM) if for any graph $G=(V, E) \in \mathcal{G}$, any $v \in V, \Lambda \subset V$ and any $\sigma_{\Lambda}, \tau_{\Lambda} \in\{0,1\}^{\Lambda}$,

$$
\left|p_{v}^{\sigma_{\Lambda}}-p_{v}^{\tau_{\Lambda}}\right| \leq \exp (-\Omega(\operatorname{dist}(v, S))),
$$

where $S \subset \Lambda$ is the subset on which $\sigma_{\Lambda}$ and $\tau_{\Lambda} \operatorname{differ}$, and $\operatorname{dist}(v, S)$ is the shortest distance from $v$ to any vertex in $S$. 
Weak spatial mixing is defined similarly by measuring the decay with respect to $\operatorname{dist}(v, \Lambda)$ instead of $\operatorname{dist}(v, S)$. Spatial mixing properties are also called correlation decay in statistical physics.

If SSM holds, then the error caused by early termination in $T_{\mathrm{SAW}}(G, v)$ and arbitrary boundary values is only exponentially small in the depth. Hence the algorithm is an FPTAS. In a lot of cases, the existence of an FPTAS boils down to establish SSM.

\subsection{The Uniqueness Condition in Regular Trees}

Let $\mathbb{T}_{d}$ denote the infinite $d$-regular tree, also known as the Bethe lattice or the Cayley tree. If we pick an arbitrary vertex as the root of $\mathbb{T}_{d}$, then the root has $d$ children and every other vertex has $d-1$ children. Notice that the difference between $\mathbb{T}_{d}$ and an infinite $(d-1)$-ary tree is only the degree of the root. We consider the uniqueness of Gibbs measures on $\mathbb{T}_{d}$, where the field is uniformly $\lambda>0$. Due to the symmetric structure of $\mathbb{T}_{d}$, the standard recursion (2) thus becomes $R_{v}=f_{d-1}\left(R_{v_{i}}\right)$ (for any vertex $v$ other than the root), where $f_{d}(x)=\lambda\left(\frac{\beta x+1}{x+\gamma}\right)^{d}$ is the symmetrized version of $F_{d}(\mathbf{x})$.

For anti-ferromagnetic systems, that is, $\beta \gamma<1$, there is a unique fixed point to $f_{d}(x)=x$, denoted by $\widehat{x}$. It has been shown that the Gibbs measure in $\mathbb{T}_{d}$ is unique if and only if $\left|f_{d-1}^{\prime}(\widehat{x})\right| \leq 1[10,5]$.

In contrast, if $\beta \gamma>1$, then $f_{d}^{\prime}(x)>0$ for any $x>0$. There may be 1 or 3 positive fixed points such that $x=f_{d}(x)$. It is known $[10,5]$ that the Gibbs measure of two-state spin systems in $\mathbb{T}_{d}$ is unique if and only if there is only one fixed point for $x=f_{d-1}(x)$, or equivalently, for all fixed points $\widehat{x}_{d}$ of $f_{d}(x), f_{d}^{\prime}\left(\widehat{x}_{d}\right)<1$.

Let $\Delta_{c}:=\frac{\sqrt{\beta \gamma}+1}{\sqrt{\beta \gamma}-1}$. Then we have the following result.

- Proposition 5. If $\Delta-1<\Delta_{c}$, then the uniqueness condition in $\mathbb{T}_{\Delta}$ holds regardless of the field.

Note that the condition $\Delta-1<\Delta_{c}$ matches the exact threshold of fast mixing for Gibbs samplers in the Ising model [15]. In Section 3.1, we will show that, SSM holds and there exists an FPTAS for the partition function, in graphs with degree bound $\Delta<\Delta_{c}+1$. This is Theorem 13.

To study general graphs, one needs to consider infinite regular trees of all degrees. If $\beta>1$ (still assuming $\beta \gamma>1$ and $\beta \leq \gamma$ ), then there is no $\lambda$ such that the uniqueness condition holds in $\mathbb{T}_{d}$ for all degrees $d \geq 2$. In contrast, let $\lambda_{c}^{i n t}:=(\gamma / \beta)^{\frac{\left\lceil\Delta_{c}\right\rceil+1}{2}}$ and we have the following.

- Proposition 6. Let $(\beta, \gamma)$ be two parameters such that $\beta \gamma>1$ and $\beta \leq 1<\gamma$. The uniqueness condition holds in $\mathbb{T}_{d}$ for all degrees $d \geq 2$ if and only if $\lambda<\lambda_{c}^{\text {int }}$.

However, there exists $(\beta, \gamma, \lambda)$ and an (irregular) tree $T$ such that $\beta \gamma>1, \beta \leq 1<\gamma$, and $\lambda<\lambda_{c}^{i n t}$ and SSM does not hold in $T$. This is discussed in Section 5. Recall that $\lambda_{c}:=(\gamma / \beta)^{\frac{\Delta_{c}+1}{2}}$. If we replace $\lambda_{c}^{i n t}$ with $\lambda_{c} \leq \lambda_{c}^{i n t}$ in the condition of Proposition 6 , that is, $\beta \gamma>1, \beta \leq 1<\gamma$, and $\lambda<\lambda_{c}$, then SSM holds in all graphs and an FPTAS exists. This is shown in Section 3.2, Theorem 18.

Details and proofs about Propositions 5 and 6 are given in Section 6.1.

\subsection{The Potential Method}

We would like to prove the strong spatial mixing in arbitrary trees, sometimes with bounded degree $\Delta$, under certain conditions. This is sufficient for approximation algorithms due to 
the self-avoiding walk tree construction. Our main technique in the analysis is the potential method. The analysis in this section is a standard routine, with some specialization to ferromagnetic 2-spin models (cf. [12, 16]). To avoid interrupting the flow, we move all details and proofs to Section 6.2.

Roughly speaking, instead of studying (2) directly, we use a potential function $\Phi(x)$ to map the original recursion to a new domain (see the commutative diagram Figure 1). Morally we can choose whatever function as the potential function. However, we would like to pick "good" ones so as to help the analysis of the contraction. Define $\varphi(x):=\Phi^{\prime}(x)$ and

$$
C_{\varphi, d}(\mathbf{x}):=\varphi\left(F_{d}(\mathbf{x})\right) \cdot \sum_{i=1}^{d}\left|\frac{\partial F_{d}}{\partial x_{i}}\right| \frac{1}{\varphi\left(x_{i}\right)} .
$$

- Definition 7. Let $\Phi: \mathbb{R}^{+} \rightarrow \mathbb{R}^{+}$be a differentiable and monotonically increasing function. Let $\varphi(x)$ and $C_{\varphi, d}(\mathbf{x})$ be defined as above. Then $\Phi(x)$ is a good potential function for degree $d$ and field $\lambda$ if it satisfies the following conditions:

1. there exists a constant $C_{1}, C_{2}>0$ such that $C_{1} \leq \varphi(x) \leq C_{2}$ for all $x \in\left[\lambda \gamma^{-d}, \lambda \beta^{d}\right]$;

2. there exists a constant $\alpha<1$ such that $C_{\varphi, d}(\mathbf{x}) \leq \alpha$ for all $x_{i} \in\left[\lambda \gamma^{-d}, \lambda \beta^{d}\right]$.

We say $\Phi(x)$ is a good potential function for $d$ and field $\pi$, if $\Phi(x)$ is a good potential function for $d$ and any $\lambda$ in the codomain of $\pi$,

In Definition 7, Condition 1 is rather easy to satisfy. The crux is in fact Condition 2. We call $\alpha$ in Condition 2 the amortized contraction ratio of $\Phi(x)$. It has the following algorithmic implication. The proof is based on establishing strong spatial mixing.

- Lemma 8. Let $(\beta, \gamma)$ be two parameters such that $\beta \gamma>1$. Let $G=(V, E)$ be a graph with a maximum degree $\Delta$ and $n$ many vertices and $\pi$ be a field on $G$. Let $\lambda=\max _{v \in V}\{\pi(v)\}$. If there exists a good potential function for $\pi$ and all $d \in[1, \Delta-1]$ with contraction ratio $\alpha<1$, then $Z_{\beta, \gamma, \pi}(G)$ can be approximated deterministically within a relative error $\varepsilon$ in time $O\left(n\left(\frac{n \lambda}{\varepsilon}\right)^{\frac{\log (\Delta-1)}{-\log \alpha}}\right)$.

When the degree is unbounded, the SAW tree may grow super polynomially even if the depth is of order $\log n$. We use a refined metric replacing the naive graph distance used in Definition 4. Strong spatial mixing under this metric is also called computationally efficient correlation decay $[11,12]$.

- Definition 9. Let $T$ be a rooted tree and $M>1$ be a constant. For any vertex $v$ in $T$, define the $M$-based depth of $v$, denoted $\ell_{M}(v)$, such that $\ell_{M}(v)=0$ if $v$ is the root, and $\ell_{M}(v)=\ell_{M}(u)+\left\lceil\log _{M}(d+1)\right\rceil$ if $v$ is a child of $u$ and $u$ has degree $d$.

Let $B(\ell)$ be the set of all vertices whose $M$-based depths of $v$ is at most $\ell$. It is easy to verify inductively such that $|B(\ell)| \leq M^{\ell}$ in a tree. We then define a slightly stronger notion of potential functions.

- Definition 10. Let $\Phi: \mathbb{R}^{+} \rightarrow \mathbb{R}^{+}$be a differentiable and monotonically increasing function. Let $\varphi(x)$ and $C_{\varphi, d}(\mathbf{x})$ defined in the same way as in Definition 7 . Then $\Phi(x)$ is a universal potential function for the field $\lambda$ if it satisfies the following conditions:

1. there are two constants $C_{1}, C_{2}>0$ such that $C_{1} \leq \varphi(x) \leq C_{2}$ for any $x \in(0, \lambda]$;

2. there exists a constant $\alpha<1$ such that for all $d, C_{\varphi, d}(\mathbf{x}) \leq \alpha^{\left\lceil\log _{M}(d+1)\right\rceil}$ for all $x_{i} \in(0, \lambda]$;

We say $\Phi(x)$ is a universal potential function for a field $\pi$, if $\Phi(x)$ is a universal potential function for any $\lambda$ in the codomain of $\pi$. We also call $\alpha$ the contraction ratio and call $M$ the 
base. The following two lemmas show that our main theorems follow from the existence of a universal potential function.

The way we define universal potential functions restricts them to only apply to the range of $(0, \lambda]$. This will be true in our applications (see for example Claim 16).

- Lemma 11. Let $(\beta, \gamma, \lambda)$ be three parameters such that $\beta \gamma>1, \beta \leq \gamma$, and $\lambda<\lambda_{c}$. Let T and $T^{\prime}$ be two trees that agree on the first $\ell$ levels with root $v$ and $v^{\prime}$ respectively. If there exists a universal potential function $\Phi(x)$, then $\left|p_{v}-p_{v^{\prime}}\right| \leq O(\exp (-\ell))$.

- Lemma 12. Let $(\beta, \gamma)$ be two parameters such that $\beta \gamma>1$ and $\beta \leq 1<\gamma$. Let $G=(V, E)$ be a graph with $n$ many vertices and $\pi$ be a field on $G$. Let $\lambda=\max _{v \in V}\{\pi(v)\}$. If there exists a universal potential function $\Phi(x)$ for $\pi$ with contraction ratio $\alpha<1$ and base $M$, then $Z_{\beta, \gamma, \pi}(G)$ can be approximated deterministically within a relative error $\varepsilon$ in time $O\left(n^{3}\left(\frac{n \lambda}{\varepsilon}\right)^{\frac{\log M}{-\log \alpha}}\right)$.

\section{Correlation Decay below $\Delta_{c}$ or $\lambda_{c}$}

In this section, we show our main results. We will first show a folklore result for bounded degree graphs with a very simple proof. Then we continue to show the main theorem regarding general graphs. We carefully choose two appropriate potential functions and then apply Lemma 8 or Lemma 12.

\subsection{Bounded Degree Graphs}

We first apply our framework to get FPTAS for graphs with degree bound $\Delta<\Delta_{c}+1=$ $\frac{2 \sqrt{\beta \gamma}}{\sqrt{\beta \gamma}-1}$. Correlation decay for graphs with such degree bounds is folklore and can be found in [14] for the Ising model. Algorithmic implications are also shown, e.g. in [20]. As we shall see, the proof is very simple in our framework. Note that $\lambda, \Delta$, and $\alpha$ are considered constants for the FPTAS.

- Theorem 13. Let $(\beta, \gamma)$ be two parameters such that $\beta \gamma>1$. Let $G=(V, E)$ be a graph with a maximum degree $\Delta<\Delta_{c}+1$ and $n$ many vertices, and let $\pi$ be a field on $G$. Let $\lambda=\max _{v \in V}\{\pi(v)\}$. Then $Z_{\beta, \gamma, \pi}(G)$ can be approximated deterministically within a relative error $\varepsilon$ in time $O\left(n\left(\frac{n \lambda}{\varepsilon}\right)^{\frac{\log (\Delta-1)}{-\log \alpha}}\right)$, where $\alpha=\frac{\Delta-1}{\Delta_{c}}$.

Proof. We choose our potential function to be $\Phi_{1}(x)=\log x$ such that $\varphi_{1}(x):=\Phi_{1}^{\prime}(x)=\frac{1}{x}$. We verify the conditions of Definition 7. Condition 1 is trivial. For Condition 2, we have that for any integer $1 \leq d \leq \Delta-1$,

$$
\begin{aligned}
C_{\varphi_{1}, d}(\mathbf{x}) & =\varphi_{1}\left(F_{d}(\mathbf{x})\right) \sum_{i=1}^{d} \frac{\partial F_{d}}{\partial x_{i}} \cdot \frac{1}{\varphi_{1}(x)} \\
& =\frac{1}{F_{d}(\mathbf{x})} \sum_{i=1}^{d} F_{d}(\mathbf{x}) \cdot \frac{\beta \gamma-1}{\left(x_{i}+\beta\right)\left(\gamma x_{i}+1\right)} \cdot x_{i} \\
& =\sum_{i=1}^{d} \frac{(\beta \gamma-1) x_{i}}{\left(\gamma x_{i}+1\right)\left(x_{i}+\beta\right)} \leq \sum_{i=1}^{d} \frac{1}{\Delta_{c}}=\frac{d}{\Delta_{c}} \leq \frac{\Delta-1}{\Delta_{c}}=\alpha
\end{aligned}
$$


where we used the fact that for any $x>0$,

$$
\frac{(\beta \gamma-1) x}{(\gamma x+1)(x+\beta)} \leq \frac{1}{\Delta_{c}}
$$

Hence $\Phi_{1}(x)$ is a good potential function for all degrees $d \in[1, \Delta-1]$ with contraction ratio $\alpha$. The theorem follows by Lemma 8 .

Note that Theorem 13 matches the uniqueness condition in Proposition 5 and, restricted to the Ising model, the fast mixing bound of Gibbs samplers in [15].

\subsection{General Graphs}

Recall that $\lambda_{c}=\left(\frac{\gamma}{\beta}\right)^{\frac{\Delta_{c}+1}{2}}=\left(\frac{\gamma}{\beta}\right)^{\frac{\sqrt{\beta \gamma}}{\sqrt{\beta \gamma}-1}}$. The following two technical lemmas show some important properties regarding the threshold $\lambda_{c}$, which are keys to get our main theorems. Proofs are given in Section 6.3.

- Lemma 14. Let $\beta, \gamma$ be two parameters such that $\beta \gamma>1$ and $\beta \leq \gamma$. For any $0<x \leq \lambda_{c}$, $\frac{\beta x+1}{x+\gamma} \leq 1$.

- Lemma 15. Let $\beta, \gamma$ be two parameters such that $\beta \gamma>1$ and $\beta \leq \gamma$. For any $0<x \leq \lambda_{c}$, we have

$$
(\beta \gamma-1) x \log \frac{\lambda_{c}}{x} \leq(\beta x+1)(x+\gamma) \log \frac{x+\gamma}{\beta x+1} .
$$

In our applications, the quantity $x$ in both lemmas will be the ratio of marginal probabilities in trees, denoted by $R_{v}$ for a vertex $v$. To make use of these properties, one key requirement is that $0<x \leq \lambda_{c}$. This is not necessarily true in trees with pinning (and therefore not true in general SAW trees). Nevertheless, it does hold in trees without pinning.

- Claim 16. For $(\beta, \gamma, \lambda)$ where $\beta \gamma>1, \beta \leq \gamma$, and $\lambda<\lambda_{c}, R_{v} \in(0, \lambda]$ holds in trees without pinning.

We prove Claim 16 by induction. For any tree $T_{v}$, if $v$ is the only vertex, then $R_{v}=\lambda$ and the base case holds. Given Lemma 14 and $\lambda<\lambda_{c}$, the inductive step to show Claim 16 follows from the standard tree recursion (2).

In addition, it also holds when $\beta \leq 1$, in trees even with pinning (but not counting the pinned vertices). This includes the SAW tree construction as special cases. To see that, for any vertex $v$, if one of $v$ 's child, say $u$, is pinned to 0 (or 1 ), then we can just remove $u$ and change the field of $v$ from $\lambda_{v}$ to $\lambda_{v}^{\prime}=\lambda_{v} \beta$ (or $\lambda_{v}^{\prime}=\lambda_{v} / \gamma$ ), without affecting the marginal probability of $v$ and any other vertices. By our assumptions $\lambda_{v}<\lambda_{c}$ and $\beta \leq 1<\gamma$, we have that $\lambda_{v}^{\prime}<\lambda_{c}$ as well. Hence, after removing all pinned vertices, we still have that $\lambda_{v} \leq \lambda_{c}$ for all $v \in V$. This reduces to Claim 16 .

Indeed, both of Theorem 1 and 2 can be generalized to the setting where vertices may have different external fields as long as they are all below $\lambda_{c}$, as follows.

- Theorem 17. Let $(\beta, \gamma)$ be two parameters such that $\beta \gamma>1, \beta \leq \gamma$, and $\lambda<\lambda_{c}$. Let $T_{v}$ and $T_{v^{\prime}}^{\prime}$ be two trees with roots $v$ and $v^{\prime}$ respectively. Let $\lambda=\max _{u \in T_{v} \cup T_{v^{\prime}}^{\prime}}\{\pi(u)\}$. If $\lambda<\lambda_{c}$ and in the first $\ell$ levels, $T_{v}$ and $T_{v^{\prime}}^{\prime}$ have the same structure and external fields for corresponding pairs of vertices, then $\left|p_{v}-p_{v^{\prime}}\right| \leq O(\exp (-\ell))$. 
- Theorem 18. Let $(\beta, \gamma)$ be two parameters such that $\beta \gamma>1$ and $\beta \leq 1<\gamma$. Let $G=(V, E)$ be a graph with $n$ many vertices, and let $\pi$ be a field on $G$. Let $\lambda=\max _{v \in V}\{\pi(v)\}$. If $\lambda<\lambda_{c}$, then $Z_{\beta, \gamma, \pi}(G)$ can be approximated deterministically within a relative error $\varepsilon$ in time $O\left(n\left(\frac{n \lambda}{\varepsilon}\right)^{\frac{\log M}{-\log \alpha}}\right)$, where $M>1$ and $\alpha<1$ are two constants depending on $(\beta, \gamma, \lambda)$.

To show Theorem 17 and Theorem 18, we will apply Lemma 11 and Lemma 12. Essentially we only need to show the existence of a universal potential function.

Let $g_{\lambda}(x):=\frac{(\beta \gamma-1) x \log \frac{\lambda}{x}}{(\beta x+1)(x+\gamma) \log \frac{x+\gamma}{\beta x+1}}$. By Lemma 15, $g_{\lambda_{c}}(x) \leq 1$. For $\lambda<\lambda_{c}$, note that $\lim _{x \rightarrow 0} g_{\lambda}(x)=0$. Hence there exists $0<\varepsilon<\lambda$ and $0<\delta<1$ such that if $0<x<\varepsilon$, $g_{\lambda}(x)<\delta$. Moreover, if $\varepsilon \leq x \leq \lambda$, then $\frac{g_{\lambda}(x)}{g_{\lambda_{c}}(x)}=\frac{\log \lambda-\log x}{\log \lambda_{c}-\log x} \leq \frac{\log \lambda-\log \varepsilon}{\log \lambda_{c}-\log \varepsilon}$. Let

$$
\alpha_{\lambda}:=\max \left\{\delta, \frac{\log \lambda-\log \varepsilon}{\log \lambda_{c}-\log \varepsilon}\right\}<1
$$

Then we have the following lemma.

Lemma 19. Let $\beta, \gamma$ be two parameters such that $\beta \gamma>1$ and $\beta \leq \gamma$. If $\lambda<\lambda_{c}$, then $g_{\lambda}(x) \leq \alpha_{\lambda}$ for any $0<x \leq \lambda$, where $\alpha_{\lambda}<1$ is defined above.

Let $t:=\frac{\alpha_{\lambda} \gamma}{\beta \gamma-1} \log \frac{\lambda+\gamma}{\beta \lambda+1}$ so that for any $0<x \leq \lambda$,

$$
t \leq \frac{\alpha_{\lambda}(\beta x+1)(x+\gamma)}{\beta \gamma-1} \log \frac{x+\gamma}{\beta x+1}
$$

We define $\varphi_{2}(x):=\min \left\{\frac{1}{t}, \frac{1}{x \log \frac{\lambda}{x}}\right\}$. To be specific, note that $x \log \frac{\lambda}{x} \leq \frac{\lambda}{e}$ for any $0<x \leq \lambda$. If $t \geq \frac{\lambda}{e}$, then $\frac{1}{x \log \frac{\lambda}{x}} \geq \frac{1}{t}$ for any $0<x \leq \lambda$. In this case, we let

$$
\varphi_{2}(x):=\frac{1}{t}
$$

Otherwise $t<\frac{\lambda}{e}$, and there are two roots to $x \log \frac{\lambda}{x}=t$ in $(0, \lambda]$. Denote them by $x_{0}$ and $x_{1}$. We define

$$
\varphi_{2}(x):= \begin{cases}\frac{1}{t} & 0 \leq x<x_{0} \\ \frac{1}{x \log \frac{\lambda}{x}} & x_{0} \leq x<x_{1} \\ \frac{1}{t} & x_{1} \leq x<\lambda\end{cases}
$$

We define $\Phi_{2}(x):=\int_{0}^{x} \varphi_{2}(y) d y$ so that $\Phi_{2}^{\prime}(x)=\varphi_{2}(x)$. By our choice of $\varphi_{2}(x)$, it always holds that for any $0<x \leq \lambda$,

$$
\varphi_{2}(x) x \log \frac{\lambda}{x} \leq 1
$$

and by Lemma 14 and Lemma 19,

$$
\frac{\beta \gamma-1}{(\beta x+1)(x+\gamma)} \cdot \frac{1}{\varphi_{2}(x)} \leq \alpha_{\lambda} \log \frac{x+\gamma}{\beta x+1} .
$$

Now, we are ready to prove Theorems 17 and 18 .

Proof of Theorems 17 and 18. We claim that $\Phi_{2}(x)$ is a universal potential function for any field $\pi$ with an upper bound $\lambda$, with contraction ratio $\alpha_{\lambda}$ given above and base $M$ that 
will be determined shortly. Theorem 17 and Theorem 18 follow from $\Phi_{2}(x)$ combined with Lemma 11 and 12, respectively. We verify the two conditions in Definition 10.

For Condition 1, it is easy to see that in case $(4), \varphi_{2}(x)=\frac{1}{t}$ for any $x \in(0, \lambda]$, and in case $(5), \frac{e}{\lambda} \leq \varphi_{2}(x) \leq \frac{1}{t}$ for any $x \in(0, \lambda]$.

For Condition 2, we have that

$$
\begin{aligned}
C_{\varphi_{2}, d}(\mathbf{x}) & =\varphi_{2}\left(F_{d}(\mathbf{x})\right) \sum_{i=1}^{d} \frac{\partial F_{d}}{\partial x_{i}} \cdot \frac{1}{\varphi_{2}\left(x_{i}\right)} \\
& =\varphi_{2}\left(F_{d}(\mathbf{x})\right) F_{d}(\mathbf{x}) \sum_{i=1}^{d} \frac{\beta \gamma-1}{\left(\beta x_{i}+1\right)\left(x_{i}+\gamma\right)} \cdot \frac{1}{\varphi_{2}\left(x_{i}\right)} \\
& \leq \varphi_{2}\left(F_{d}(\mathbf{x})\right) F_{d}(\mathbf{x}) \sum_{i=1}^{d} \alpha_{\lambda} \log \frac{x_{i}+\gamma}{\beta x_{i}+1} \\
& =\alpha_{\lambda} \varphi_{2}\left(F_{d}(\mathbf{x})\right) F_{d}(\mathbf{x}) \log \frac{\lambda}{F_{d}(\mathbf{x})} \\
& \leq \alpha_{\lambda} .
\end{aligned}
$$

Moreover, $F_{d}(\mathbf{x})<\lambda\left(\frac{\beta \lambda+1}{\lambda+\gamma}\right)^{d}$ for any $x_{i} \in(0, \lambda]$, and $\frac{\beta \lambda+1}{\lambda+\gamma}<1$ by Lemma 14 . Then there exists $d_{0} \geq 1$ such that $\left(\frac{\beta \lambda+1}{\lambda+\gamma}\right)^{d_{0}}<e^{-1}$. Hence, for any $d>d_{0}$,

$$
\begin{aligned}
C_{\varphi_{2}, d}(\mathbf{x}) & \leq \frac{\alpha_{\lambda}}{t} F_{d}(\mathbf{x}) \log \frac{\lambda}{F_{d}(\mathbf{x})} \\
& \leq \frac{\alpha_{\lambda} \lambda}{t}\left(\frac{\beta \lambda+1}{\lambda+\gamma}\right)^{d} d \log \frac{\beta \lambda+1}{\lambda+\gamma}
\end{aligned}
$$

Therefore, there exists an integer $M \geq d_{0}$ such that for any $1 \leq d<M, C_{\varphi_{2}, d}(\mathbf{x}) \leq$ $\alpha_{\lambda} \leq \alpha_{\lambda}^{\left\lceil\log _{M}(d+1)\right\rceil}$ and for any $d \geq M, C_{\varphi_{2}, d}(\mathbf{x}) \leq \frac{\alpha_{\lambda} \lambda}{t}\left(\frac{\beta \lambda+1}{\lambda+\gamma}\right)^{d} d \log \left(\frac{\beta \lambda+1}{\lambda+\gamma}\right) \leq \alpha_{\lambda}^{\left\lceil\log _{M}(d+1)\right\rceil}$. Condition 2 holds.

\subsection{Heuristics behind $\Phi_{2}(x)$}

The most intricate part of our proofs of Theorem 17 and Theorem 18 is the choice of the potential function $\Phi_{2}(x)$ given by (5). Here we give a brief heuristic of deriving it. It is more of an "educated guess" than a rigorous argument.

We want to pick $\Phi_{2}(x)$ such that Condition 2 holds. In particular, we want

$$
\varphi_{2}\left(F_{d}(\mathbf{x})\right) \sum_{i=1}^{d} \frac{\partial F_{d}}{\partial x_{i}} \cdot \frac{1}{\varphi_{2}\left(x_{i}\right)}<1 .
$$

It is fair to assume that the left hand side of the equation above takes its maximum when all $x_{i}$ 's are equal. Hence, we hope the following to hold

$$
\frac{\varphi_{2}\left(f_{d}(x)\right) f_{d}^{\prime}(x)}{\varphi_{2}(x)}<1
$$

where $f_{d}(x)=\lambda\left(\frac{\beta x+1}{x+\gamma}\right)^{d}$ is the symmetrized version of $F_{d}(\mathbf{x})$. We will use $z:=f_{d}(x)$ to simplify notation. Since we want (8) to hold for all degrees $d$, we hope to eliminate $d$ from 
the left hand side of (8). Notice that $\varphi_{2}(x)$ should be independent from $d$. Therefore, we take the derivative of $\varphi_{2}\left(f_{d}(x)\right) f_{d}^{\prime}(x)$ against $d$ and get

$$
\begin{aligned}
\frac{\partial \varphi_{2}\left(f_{d}(x)\right) f_{d}^{\prime}(x)}{\partial d} & =\frac{\beta \gamma-1}{(\beta x+1)(x+\gamma)}\left(\varphi_{2}(z) z+\varphi_{2}(z) z \log \frac{z}{\lambda}+\varphi_{2}^{\prime}(z) z^{2} \log \frac{z}{\lambda}\right) \\
& =\frac{(\beta \gamma-1) z \varphi_{2}(z)}{(\beta x+1)(x+\gamma)}\left(1+\log \frac{z}{\lambda}+\left(\log \varphi_{2}(z)\right)^{\prime} z \log \frac{z}{\lambda}\right) .
\end{aligned}
$$

We may achieve our goal of eliminating $d$ by imposing the sum in the last parenthesis to be 0 , namely

$$
\begin{aligned}
\left(\log \varphi_{2}(z)\right)^{\prime} & =-\frac{1}{z}-\frac{1}{z \log \frac{z}{\lambda}} \\
& =-(\log z)^{\prime}-\left(\log \log \frac{\lambda}{z}\right)^{\prime} .
\end{aligned}
$$

From (9), it is easy to see that $\varphi_{2}(z)=\frac{1}{z \log \frac{\lambda}{z}}$ satisfies our need. To get the full definition of (5), we apply a thresholding trick to bound $\varphi_{2}(z)$ away from 0 .

\subsection{Discussion of the $\beta>1$ case}

We cannot combine conditions of Theorem 17 and Theorem 18 together to have an FPTAS. In particular, when $\beta>1$ strong special mixing fails for any $\lambda$ even if $\lambda<\lambda_{c}$. To see this, given a $\Delta$-ary tree $T$, we can append $t$ many children to every vertex in $T$ to get a new tree $T^{\prime}$ and impose a partial configuration $\sigma$ where all these new children are pinned to 0 . Effectively, the tree $T^{\prime}$ is equivalent to $T$ where every vertex has a new external field of $\lambda \beta^{t}$, which is larger than $\lambda_{c}^{i n t}$ if $t$ is sufficiently large regardless of $\lambda$. Then by Proposition 6 , long range correlation exists in $T^{\prime}$ with the partial configuration $\sigma$, and strong spatial mixing fails.

On the other hand, it is easy to see from the proof that, Theorem 17 can be generalized to allow a partial configuration $\sigma$ on some subset $\Lambda$ where the marginal probability of every vertex $v \in \Lambda$ satisfies $p_{v}^{\sigma} \leq \frac{\lambda_{c}}{\lambda_{c}+1}$. This is not the case for the SAW tree which our algorithm relies on when $\beta>1$. However, the following observation shows that if $\lambda_{v} \leq \lambda_{c} \leq \frac{\gamma-1}{\beta-1}$, then the marginal probability of any instance $G$ satisfies this requirement. Thus, it seems the only piece missing to obtain an algorithm is to design a better recursion tree instead of the SAW tree.

- Proposition 20. Let $(\beta, \gamma)$ be two parameters such that $1 \leq \beta \leq \gamma$ and $\beta \gamma>1$. Let $\lambda \leq \frac{\gamma-1}{\beta-1}$ be another parameter. For any graph $G=(V, E)$, if $\pi(v) \leq \lambda$ for all $v \in V$, then $p_{v} \leq \frac{\lambda}{\lambda+1}$.

To prove this proposition, we need to use the random cluster formulation of 2-spin models. Let $G$ be a graph and $e=\left(v_{1}, v_{2}\right)$ be one of its edges. Let $G^{+}$be the graph where the edge $e$ is contracted, and $G^{-}$be the graph where $e$ is removed. Moreover, in $G^{+}$, we assign $\pi^{+}(\widetilde{v})=\lambda_{v_{1}} \lambda_{v_{2}} \frac{\beta-1}{\gamma-1}$, where $\widetilde{v}$ is the vertex obtained from contacting $e$. Then we have that

$$
Z(G)=Z\left(G^{-}\right)+(\gamma-1) Z\left(G^{+}\right)
$$

where we write $Z(G)$ instead of $Z_{\beta, \gamma, \pi}(G)$ to simplify the notation. To show the equation above we only need a simple adapation of the random cluster formulation of the Ising model to the 2-spin setting. 
Proof of Proposition 20. Suppose $G=(V, E)$ where $|V|=n$ and $|E|=m$. We show the claim by inducting on $(m, n)$. Clearly the statement holds when $m=0$ or $n=1$. Hence we may assume the claim holds for $\left(m^{\prime}, n\right)$ where $m^{\prime}<m$ as well as $\left(m^{\prime}, n^{\prime}\right)$ where $n^{\prime}<n$, and show that the claim holds for $(m, n)$.

Pick an arbitrary edge $e=\left(v_{1}, v_{2}\right)$ in $G$. Let $G^{+}$and $G^{-}$be as in the random cluster formulation. It is easy to see that $\pi(\widetilde{v})=\lambda_{v_{1}} \lambda_{v_{2}} \frac{\beta-1}{\gamma-1} \leq \lambda$. Hence both $G^{+}$and $G^{-}$satisfy the induction hypothesis. It implies that $p_{G^{-} ; v} \leq \frac{\lambda}{\lambda+1}$ for any $v$, where $p_{G^{-} ; v}$ is the mariginal probability of $v$ in $G^{-}$. Moreover, $p_{G^{+} ; v} \leq \frac{\lambda}{\lambda+1}$ for any $v \in V^{+}$, where $V^{+}$is the vertex set of $G^{+}$. Let $\delta$ be a mapping $V \rightarrow V^{+}$such that $\delta(v)=v$ if $v \neq v_{1}, v_{2}$ and $\delta\left(v_{1}\right)=\delta\left(v_{2}\right)=\widetilde{v}$. Then using (10) we have that for any vertex $v \in V$,

$$
\begin{aligned}
p_{G ; v} & =\frac{Z^{\sigma(v)=0}(G)}{Z(G)}=\frac{Z^{\sigma(v)=0}\left(G^{-}\right)+(\gamma-1) Z^{\sigma(\delta(v))=0}\left(G^{+}\right)}{Z\left(G^{-}\right)+(\gamma-1) Z\left(G^{+}\right)} \\
& =p_{G^{-} ; v} \cdot \frac{Z\left(G^{-}\right)}{Z\left(G^{-}\right)+(\gamma-1) Z\left(G^{+}\right)}+p_{G^{+} ; \delta(v)} \cdot \frac{(\gamma-1) Z\left(G^{+}\right)}{Z\left(G^{-}\right)+(\gamma-1) Z\left(G^{+}\right)} \\
& \leq \frac{\lambda}{\lambda+1} \cdot \frac{Z\left(G^{-}\right)}{Z\left(G^{-}\right)+Z\left(G^{+}\right)}+\frac{\lambda}{\lambda+1} \cdot \frac{(\gamma-1) Z\left(G^{+}\right)}{Z\left(G^{-}\right)+(\gamma-1) Z\left(G^{+}\right)}=\frac{\lambda}{\lambda+1},
\end{aligned}
$$

where in the last line we use the induction hypotheses.

\section{Correlation Decay Beyond $\lambda_{c}$}

Let $\beta, \gamma$ be two parameters such that $\beta \leq 1<\gamma$ and $\beta \gamma>1$. In this section we give an example to show that if $\Delta_{c}$ is not an integer, then correlation decay still holds for a small interval beyond $\lambda_{c}$. To simplify the presentation, we assume that $\pi$ is a uniform field such that $\pi(v)=\lambda$. Note that the potential function $\varphi_{2}(x)$ does not extend beyond $\lambda_{c}$.

Let $\beta=0.6$ and $\gamma=2$. Then $\Delta_{c}=\frac{\sqrt{\beta \gamma}+1}{\sqrt{\beta \gamma}-1} \approx 21.95$ and $\lambda_{c}=(\gamma / \beta)^{\frac{\Delta_{c}+1}{2}}<1002761$. Let $\lambda=1002762>\lambda_{c}$. We will show that $\# 2 \operatorname{SPIN}(\beta, \gamma, \lambda)$ still has an FPTAS.

Define a constant $t$ as

$$
t:=\frac{\sqrt{\beta \gamma}+1}{\sqrt{\beta \gamma}-1} \cdot \frac{\log \sqrt{\gamma / \beta}}{\sqrt{\gamma / \beta}+1}-\log (1+\sqrt{\beta / \gamma}) \approx 4.24032
$$

We consider the potential function $\Phi_{3}(x)$ so that $\varphi_{3}(x):=\frac{1}{x(\log (1+1 / x)+t)}$. With this choice,

$$
\begin{aligned}
C_{\varphi_{3}, d}(\mathbf{x}) & =\varphi_{3}\left(F_{d}(\mathbf{x})\right) \sum_{i=1}^{d} \frac{\partial F_{d}}{\partial x_{i}} \cdot \frac{1}{\varphi_{3}(x)} \\
& =\frac{\beta \gamma-1}{\log \left(1+1 / F_{d}(\mathbf{x})\right)+t} \sum_{i=1}^{d} \frac{x_{i}\left(\log \left(1+1 / x_{i}\right)+t\right)}{\left(\beta x_{i}+1\right)\left(x_{i}+\gamma\right)} .
\end{aligned}
$$

We do a change of variables. Let $r_{i}=\frac{\beta x_{i}+1}{x_{i}+\gamma}$. Then $x_{i}=\frac{\gamma r_{i}-1}{\beta-r_{i}}, \beta x_{i}+1=\frac{r_{i}(\beta \gamma-1)}{\beta-r_{i}}$, and $x_{i}+\gamma=\frac{\beta \gamma-1}{\beta-r_{i}}$. Hence,

$$
\begin{aligned}
\sum_{i=1}^{d} \frac{x_{i}\left(\log \left(1+1 / x_{i}\right)+t\right)}{\left(\beta x_{i}+1\right)\left(x_{i}+\gamma\right)} & =\sum_{i=1}^{d} \frac{\left(\gamma r_{i}-1\right)\left(\beta-r_{i}\right)}{r_{i}(\beta \gamma-1)^{2}} \cdot\left(\log \left(1+\frac{\beta-r_{i}}{\gamma r_{i}-1}\right)+t\right) \\
& =\frac{1}{(\beta \gamma-1)^{2}} \sum_{i=1}^{d}\left(1+\beta \gamma-\frac{\beta}{r_{i}}-\gamma r_{i}\right)\left(\log \left(1+\frac{\beta-r_{i}}{\gamma r_{i}-1}\right)+t\right) .
\end{aligned}
$$


Furthermore, let $s_{i}=\log r_{i}$. As $r_{i} \in\left(\frac{1}{\gamma}, \beta\right), s_{i} \in(-\log \gamma, \log \beta)$. Let

$$
\rho(x):=\left(1+\beta \gamma-\beta e^{-x}-\gamma e^{x}\right)\left(\log \left(1+\frac{\beta-e^{x}}{\gamma e^{x}-1}\right)+t\right) .
$$

Then $\rho(x)$ is concave for any $x \in(-\log \gamma, \log \beta)$. It can be easily verified, as the second derivative is

$$
\begin{aligned}
\rho^{\prime \prime}(x)= & \frac{(\beta+1)(\beta \gamma-1)}{\beta-1+e^{x}(\gamma-1)}+\frac{\gamma(\beta \gamma-1)}{\gamma-1}-\frac{\beta(\beta \gamma-1)}{\beta-e^{x}}-\frac{(\beta-1)(\beta \gamma-1)^{2}}{(\gamma-1)\left(\beta-1+e^{x}(\gamma-1)\right)^{2}} \\
& -\beta t e^{-x}-\gamma t e^{x}-e^{-x}\left(\beta+e^{2 x} \gamma\right) \log \left(1+\frac{\gamma e^{x}-1}{\beta-e^{x}}\right) . \\
\leq & \gamma(\beta+1)+\frac{\gamma(\beta \gamma-1)}{\gamma-1}-\beta \gamma-\frac{\beta-1}{\gamma-1}-2 t<-5.68<0,
\end{aligned}
$$

where in the last line we used (11) and the fact that $1 / \gamma \leq e^{x} \leq \beta$. Hence, by concavity, we have that for any $x_{i} \in(0, \lambda]$,

$$
\begin{aligned}
C_{\varphi_{3}, d}(\mathbf{x}) & =\frac{\beta \gamma-1}{\log \left(1+1 / F_{d}(\mathbf{x})\right)+t} \sum_{i=1}^{d} \frac{x_{i}\left(\log \left(1+1 / x_{i}\right)+t\right)}{\left(\beta x_{i}+1\right)\left(x_{i}+\gamma\right)} \\
& \leq \frac{\beta \gamma-1}{\log \left(1+1 / f_{d}(\widetilde{x})\right)+t} \cdot \frac{d \widetilde{x}\left(\log \left(1+\widetilde{x}^{-1}\right)+t\right)}{(\beta \widetilde{x}+1)(\widetilde{x}+\gamma)}=c_{\varphi_{3}, d}(\widetilde{x}),
\end{aligned}
$$

where $\widetilde{x}>0$ is the unique solution such that $f_{d}(\widetilde{x})=F_{d}(\mathbf{x})$.

Next we show that there exists an $\alpha<1$ such that for any integer $d$ and $x>0$, $c_{\varphi_{3}, d}(x)<\alpha$. In fact, by (11), our choice of $t$, it is not hard to show that the maximum of $c_{\varphi_{3}, d}(x)$ is achieved at $x=\sqrt{\gamma / \beta}$ and $d=\Delta_{c}$, which is 1 if $\lambda=\lambda_{c}$ and is larger than 1 if $\lambda>\lambda_{c}$. However, since the degree $d$ has to be an integer, we can verify that for any integer $1 \leq d \leq 100$, the maximum of $c_{\varphi_{3}, d}(x)$ is $c_{\varphi_{3}, 22}\left(x_{22}\right)=0.999983$ where $x_{22} \approx 1.83066$. If $d>100$, then

$$
\begin{aligned}
c_{\varphi_{3}, d}(x) & =\frac{d(\beta \gamma-1)}{\log \left(1+1 / f_{d}(x)\right)+t} \cdot \frac{x\left(\log \left(1+x^{-1}\right)+t\right)}{(\beta x+1)(x+\gamma)} \\
& \leq C_{0} \cdot C_{1}<1
\end{aligned}
$$

where $C_{0}<1.07191$ is the maximum of $\frac{x\left(\log \left(1+x^{-1}\right)+t\right)}{(\beta x+1)(x+\gamma)}$ for any $x>0$, and $C_{1}<0.481875$ is the maximum of $\frac{d(\beta \gamma-1)}{\log \left(1+\lambda^{-1} \beta^{-d}\right)+t}$ for any $d>100$. Then, due to (13), we have that for any $x_{i} \in(0, \lambda], C_{\varphi_{3}, d}(\mathbf{x})<\alpha=0.999983<1$. This is the counterpart of $C_{\varphi_{2}, d}(\mathbf{x})<\alpha_{\lambda}$ in the proof of Theorem 18. To make $\varphi_{3}(x)$ satisfy Condition 1 and Condition 2 in Definition 10, it is sufficient to do a simple "chop-off" trick to $\varphi_{3}(x)$ as in (5). We will omit the detail here.

- Proposition 21. For $\beta=0.6, \gamma=2$, and $\lambda=1002762>\lambda_{c}$, \#2SPIN $(\beta, \gamma, \lambda)$ has an FPTAS.

It is easy to see that the argument above works for any $\beta \leq 1<\gamma$ and $\beta \gamma>1$ except (12), the concavity of $\rho(x)$. Indeed, the concavity does not hold if, say, $\beta=1$ and $\gamma=2$. Nevertheless, the key point here is that $\lambda_{c}$ is not the tight bound for FPTAS. Short of a conjectured optimal bound, we did not try to optimize the potential function nor the applicable range of the proof above. 


\section{Limitations of Correlation Decay}

In this section, we discuss some limitations of approximation algorithms for ferromagnetic 2-spin models based on correlation decay analysis.

The problem of counting independent sets in bipartite graphs (\#BIS) plays an important role in classifying approximate counting complexity. \#BIS is not known to have any efficient approximation algorithm, despite many attempts. However there is no known approximation preserving reduction (AP-reduction) to reduce \#BIS from \#SAT either. It is conjectured to have intermediate approximation complexity, and in particular, to have no FPRAS [3].

Goldberg and Jerrum [6] showed that for any $\beta \gamma>1$, approximating \#2SPIN $(\beta, \gamma,(0, \infty))$ can be reduced to approximating \#BIS. This is the (approximation) complexity upper bound of all ferromagnetic 2 -spin models. In contrast, by Theorem $13, \# \Delta-2 \operatorname{SPIN}(\beta, \gamma,(0, \infty))$ has an FPTAS, if $\Delta<\Delta_{c}+1$. Note that when we write $\# 2 \operatorname{SPIN}(\beta, \gamma,(0, \infty))$ the field is implicitly assumed to be at most polynomial in size of the graph (or in unary).

We then consider fields with some constant bounds. Recall that $\lambda_{c}^{i n t}=(\gamma / \beta)^{\frac{\left\lceil\Delta_{c}\right\rceil+1}{2}}$. Let $\lambda_{c}^{i n t^{\prime}}=(\gamma / \beta)^{\frac{\left\lfloor\Delta_{c}\right\rfloor+2}{2}}$. Then $\lambda_{c}^{i n t^{\prime}}=\lambda_{c}^{i n t}$ unless $\Delta_{c}$ is an integer. By reducing to antiferromagnetic 2-spin models in bipartite graphs, we have the following hardness result, which is first observed in [13, Theorem 3].

- Proposition 22. Let $(\beta, \gamma, \lambda)$ be a set of parameters such that $\beta<\gamma, \beta \gamma>1$, and $\lambda>\lambda_{c}^{\text {int }}{ }^{\prime}$. Then \#2SPIN $(\beta, \gamma,(0, \lambda])$ is \#BIS-hard.

The reduction goes as follows. Anti-ferromagnetic Ising models with a constant non-trivial field in bounded degree bipartite graphs are \#BIS-hard, if the uniqueness condition fails [1]. Given such an instance, we may first flip the truth table of one side. This effectively results in a ferromagnetic Ising model in the same bipartite graph, with two different fields on each side. By a standard diagonal transformation, we can transform such an Ising model to any ferromagnetic 2-spin model, with various local fields depending on the degree. It can be verified that for any $\lambda>\lambda_{c}^{i n t^{\prime}}$, we may pick a field in the anti-ferromagnetic Ising model to start with, such that uniqueness fails and after the transformation, the largest field in use is at most $\lambda$.

The hardness bound in Proposition 22 matches the failure of uniqueness due to Proposition 6 , unless $\Delta_{c}$ is an integer. In contrast to Proposition 22, Theorem 18 implies that if $\beta \leq 1<\gamma$ and $\lambda<\lambda_{c}=(\gamma / \beta)^{\frac{\Delta_{c}+1}{2}}$, then $\# 2 \operatorname{SPIN}(\beta, \gamma,(0, \lambda])$ has an FPTAS. Hence Theorem 18 is almost optimal, up to an integrality gap.

We note that $\lambda_{c}$ is not the tight bound for FPTAS, as observed in Proposition 21. Since the degree $d$ has to be an integer, with an appropriate choice of the potential function, there is a small interval beyond $\lambda_{c}$ such that strong spatial mixing still holds. Interestingly, it seems that $\lambda_{c}^{i n t}$ is not the right bound either. Let us make a concrete example. Let $\beta=1$ and $\gamma=2$. Then $\Delta_{c}=\frac{\sqrt{\beta \gamma}+1}{\sqrt{\beta \gamma}-1}=\frac{\sqrt{2}+1}{\sqrt{2}-1} \approx 5.82843$. Hence $\lambda_{c} \approx 10.6606$ and $\lambda_{c}^{i n t}=(2)^{\frac{6+1}{2}} \approx 11.3137$. However, even if $\lambda<\lambda_{c}^{i n t}$, the system may not exhibit spatial mixing, neither in the strong nor in the weak sense.

In fact, even the spatial mixing in the sense of Theorem 1 does not necessarily hold if $\lambda<\lambda_{c}^{i n t}$. To see this, we take any $\lambda \in[10.9759,10.9965]$ so that $\lambda_{c}<\lambda<\lambda_{c}^{i n t}$. Consider an infinite tree where at even layers, each vertex has 5 children, and at odd layers, each vertex has 7 children. There are more than one Gibbs measures in this tree. This can be easily verified from the fact that the two layer recursion function $f_{5}\left(f_{7}(x)\right)$ has three fixed points such that $x=f_{5}\left(f_{7}(x)\right)$. In addition, all three fixed points $\widehat{x}_{i}$ satisfy that $\widehat{x}_{i}<\lambda_{c}$ for $i=1,2,3$. Consider a tree $T$ with alternating degrees 5 and 7 of depth $2 \ell$, and another 
tree $T^{\prime}$ of the same structure in the first $2 \ell$ layers as $T$ but with one more layer where each vertex has, say, 50 children. It is not hard to verify that as $\ell$ increases, the marginal ratio at the root of $T$ converges to $\widehat{x}_{3}$, but the ratio at the root of $T^{\prime}$ converges to $\widehat{x}_{1}$. This example indicates that one should not expect correlation decay algorithms to work all the way up to $\lambda_{c}^{i n t}$.

At last, if we consider the uniform field case $\# 2 \operatorname{SPIN}(\beta, \gamma, \lambda)$, then our tractability results still holds. However, to extend the hardness results as in Proposition 22 from an interval of fields to a uniform one, there seems to be some technical difficulty. Suppose we want to construct a combinatorial gadget to effectively realize another field. There is a gap between $\lambda$ and the next largest possible field to realize. This is why in [13], there are some extra conditions transiting from an interval of fields to the uniform case. The observation above about the failure of SSM in irregular trees may suggest a random bipartite construction of uneven degrees. However, to analyze such a gadget is beyond the scope of the current paper.

\section{Missing Proofs}

At last, we gather technical details and proofs that are omitted in Section 2.2, Section 2.3, and Section 3.2.

\subsection{Details about the Uniqueness Threshold}

We want to prove Propositions 5 and Proposition 6. Technically by only considering the symmetric recursion $f_{d}(x)=\lambda\left(\frac{\beta x+1}{x+\gamma}\right)^{d}$, we are implicitly assuming uniform boundary conditions. If there are more than one fixed points for $f_{d}(x)$, then clearly there are multiple Gibbs measures. Hence, $f_{d}(x)$ having only one fixed point is a necessary condition for the uniqueness condition in $\mathbb{T}_{d+1}$. Moreover, it is also sufficient. The reason is that the influence on the root of an arbitrary boundary condition is bounded between those of the all " 0 " and all "1" boundary conditions.

First do some calculation here. Take the derivative of $f_{d}(x)$ :

$$
f_{d}^{\prime}(x)=\frac{d(\beta \gamma-1) f_{d}(x)}{(\beta x+1)(x+\gamma)} .
$$

Then take the second derivative:

$$
f_{d}^{\prime \prime}(x)=\frac{f_{d}^{\prime}(x)}{f_{d}(x)}-\frac{\beta}{\beta x+1}-\frac{1}{x+\gamma}=\frac{d(\beta \gamma-1)-\beta \gamma-1-2 \beta x}{(\beta x+1)(x+\gamma)} .
$$

Therefore, at $x^{*}:=\frac{d(\beta \gamma-1)-(\beta \gamma+1)}{2 \beta}, f_{d}^{\prime \prime}\left(x^{*}\right)=0$. It's easy to see when $d<\frac{\beta \gamma+1}{\beta \gamma-1}, f_{d}^{\prime \prime}(x)<0$ for all $x>0$. So $f_{d}(x)$ is concave and therefore has only one fixed point.

Since $f_{d}(x)$ has only one inflection point, there are at most three fixed points. Moreover, the uniqueness condition is equivalent to say that for all fixed points $\widehat{x}_{d}$ of $f_{d}(x), f_{d}^{\prime}\left(\widehat{x}_{d}\right)<1$. For a fixed point $\widehat{x}_{d}$, we plug it in (14):

$$
f_{d}^{\prime}\left(\widehat{x}_{d}\right)=\frac{d(\beta \gamma-1) \widehat{x}_{d}}{\left(\beta \widehat{x}_{d}+1\right)\left(\widehat{x}_{d}+\gamma\right)} .
$$

Recall that $\Delta_{c}:=\frac{\sqrt{\beta \gamma}+1}{\sqrt{\beta \gamma}-1}$. If $d<\Delta_{c}$, we have that for any $x$,

$$
\begin{aligned}
(\beta x+1)(x+\gamma)-d(\beta \gamma-1) x & =\beta x^{2}+((\beta \gamma+1)-d(\beta \gamma-1)) x+\gamma \\
& >\beta x^{2}+\left(\beta \gamma+1-(\sqrt{\beta \gamma}+1)^{2}\right) x+\gamma \\
& =(\sqrt{\beta} x-\sqrt{\gamma})^{2} \geq 0
\end{aligned}
$$


Hence $(\beta x+1)(x+\gamma)>d(\beta \gamma-1) x$. In particular, $f_{d}^{\prime}\left(\widehat{x}_{d}\right)<1$ for any fixed point $\widehat{x}_{d}$ and the uniqueness condition holds. This proves Proposition 5 .

To show Proposition 6, we may assume that $d \geq \Delta_{c}$. We may also assume that $\beta \leq \gamma$. The equation $(\beta x+1)(\gamma+x)=d(\beta \gamma-1) x$ has two solutions, which are

$$
\begin{aligned}
x_{0} & =x^{*}-\frac{\sqrt{((\beta \gamma+1)-d(\beta \gamma-1))^{2}-4 \beta \gamma}}{2 \beta} \\
\text { and } \quad x_{1} & =x^{*}+\frac{\sqrt{((\beta \gamma+1)-d(\beta \gamma-1))^{2}-4 \beta \gamma}}{2 \beta} .
\end{aligned}
$$

Notice that both of them are positive since $x_{0}+x_{1}=2 x^{*}>0$ and $x_{0} x_{1}=\beta / \gamma$.

We show that $f_{d}\left(x_{0}\right)>x_{0}$ or $f_{d}\left(x_{1}\right)<x_{1}$ is equivalent to the uniqueness condition. First we assume this condition doesn't hold, that is $f_{d}\left(x_{0}\right) \leq x_{0}$ and $f_{d}\left(x_{1}\right) \geq x_{1}$. If any of the equation holds, then $x_{0}$ or $x_{1}$ is a fixed point and the derivative is 1 . So we have non-uniqueness. Otherwise, we have $f_{d}\left(x_{0}\right)<x_{0}$ and $f_{d}\left(x_{1}\right)>x_{1}$. Since $x_{0}<x_{1}$, there is some fixed point $\widetilde{x}$ satisfying $f_{d}(\widetilde{x})=\widetilde{x}$ and $x_{0}<\widetilde{x}<x_{1}$. The second inequality implies that $(\beta \widetilde{x}+1)(\widetilde{x}+\gamma)<d(\beta \gamma-1) \widetilde{x}$. Therefore $f_{d}^{\prime}(\widetilde{x})>1$ and non-uniqueness holds.

To show the other direction, if $f_{d}\left(x_{0}\right)>x_{0}$, then

$$
f_{d}^{\prime}\left(x_{0}\right)=\frac{d(\beta \gamma-1) f\left(x_{0}\right)}{\left(\beta x_{0}+1\right)\left(x_{0}+\gamma\right)}>\frac{d(\beta \gamma-1) x_{0}}{\left(\beta x_{0}+1\right)\left(x_{0}+\gamma\right)}=1 .
$$

Assume for contradiction that $f_{d}(x)$ has three fixed points, denoted by $\widetilde{x}_{0}<\widetilde{x}_{1}<\widetilde{x}_{2}$. Then the middle fixed point $\widetilde{x}_{1}$ satisfies $f_{d}^{\prime}\left(\widetilde{x}_{1}\right)>1$. Therefore $\widetilde{x}_{1}>x_{0}$ and there are two fixed points larger than $x_{0}$. However, for $x_{0}<x \leq x^{*}, f_{d}^{\prime}(x)>1$ and $f_{d}\left(x_{0}\right)>x_{0}$. Hence there is no fixed point in this interval. For $x>x^{*}$, the function is concave and has exactly one fixed point. So there is only 1 fixed point larger than $x_{0}$. Contradiction. The case that $f_{d}\left(x_{1}\right)<x_{1}$ is similar.

These two conditions could be rewritten as

$$
\lambda>\frac{x_{0}\left(x_{0}+\gamma\right)^{d}}{\left(\beta x_{0}+1\right)^{d}}
$$

and

$$
\lambda<\frac{x_{1}\left(x_{1}+\gamma\right)^{d}}{\left(\beta x_{1}+1\right)^{d}} .
$$

Notice that the right hand side has nothing to do with $\lambda$ in both (15) and (16).

We want to see how conditions (15) and (16) change as $d$ changes. Treat $d$ as a continuous variable. Define

$$
g_{i}(d):=\frac{x_{i}\left(x_{i}+\gamma\right)^{d}}{\left(\beta x_{i}+1\right)^{d}} .
$$

where $i=0,1$ and $x_{i}$ is defined above depending on $\beta, \gamma$ and $d$. Take the derivative:

$$
\begin{aligned}
\frac{g_{i}^{\prime}(d)}{g_{i}(d)} & =\frac{\partial x_{i}}{\partial d}\left(\frac{1}{x_{i}}+\frac{d}{x_{i}+\gamma}-\frac{d \beta}{\beta x_{i}+1}\right)+\log \left(x_{i}+\gamma\right)-\log \left(\beta x_{i}+1\right) \\
& =\frac{\partial x_{i}}{\partial d}\left(\frac{1}{x_{i}}+\frac{d(1-\beta \gamma)}{\left(x_{i}+\gamma\right)\left(\beta x_{i}+1\right)}\right)+\log \frac{x_{i}+\gamma}{\beta x_{i}+1} \\
& =\frac{\partial x_{i}}{\partial d}\left(\frac{1}{x_{i}}-\frac{1}{x_{i}}\right)+\log \frac{x_{i}+\gamma}{\beta x_{i}+1}=\log \frac{x_{i}+\gamma}{\beta x_{i}+1}
\end{aligned}
$$


If $\beta \leq 1$ these two functions are increasing in $d$. Recall that $\Delta_{c}=\frac{\sqrt{\beta \gamma}+1}{\sqrt{\beta \gamma}-1}$, and $\lambda_{c}^{i n t}=$ $g_{1}\left(\left\lceil\Delta_{c}\right\rceil\right)=(\gamma / \beta)^{\frac{\left\lceil\Delta_{c}+1\right\rceil}{2}}$. Thus if $\lambda<\lambda_{c}^{i n t},(16)$ holds for all integers $d$. On the other hand,

$$
\begin{aligned}
g_{0}(d) & =\frac{x_{0}\left(x_{0}+\gamma\right)^{d}}{\left(\beta x_{0}+1\right)^{d}}>x_{0} \beta^{-d}=\frac{\beta}{\gamma x_{1}} \beta^{-d}>\frac{\beta}{\gamma 2 x^{*}} \beta^{-d} \\
& =\frac{\beta^{2}}{\gamma(d(\beta \gamma-1)-(\beta \gamma+1))} \cdot \beta^{-d} \\
& \rightarrow \infty \text { as } d \text { goes to } \infty .
\end{aligned}
$$

Hence there is no $\lambda$ such that (15) holds for all integers $d$. This proves Proposition 6 .

If $\beta>1$, then neither (15) nor (16) can hold for all integers $d$. The reason is

$$
\begin{aligned}
g_{0}(d) & =\frac{x_{0}\left(x_{0}+\gamma\right)^{d}}{\left(\beta x_{0}+1\right)^{d}}=\frac{x_{0}\left(x_{0}+\gamma\right)^{2 d}}{\left(d(\beta \gamma-1) x_{0}\right)^{d}}>x_{0}\left(\frac{\gamma}{d(\beta \gamma-1) x_{0}}\right)^{d} \\
& \rightarrow \infty \text { as } d \text { goes to } \infty
\end{aligned}
$$

as $d(\beta \gamma-1) x_{0}<\gamma$ for sufficiently large $d$, and

$$
\begin{aligned}
g_{1}(d) & =\frac{x_{1}\left(x_{1}+\gamma\right)^{d}}{\left(\beta x_{1}+1\right)^{d}}=\frac{x_{1}\left(d(\beta \gamma-1) x_{1}\right)^{d}}{\left(\beta x_{1}+1\right)^{2 d}}<x_{1}\left(\frac{d(\beta \gamma-1)}{\beta^{2} x_{1}}\right)^{d} \\
& \rightarrow 0 \text { as } d \text { goes to } \infty
\end{aligned}
$$

as $\beta^{2} x_{1}>d(\beta \gamma-1)$ for sufficiently large $d$.

\subsection{Details about the Potential Method}

In this section we provide missing details and proofs in Section 2.3.

To study correlation decay on trees, we use the standard recursion given in (2). Recall that $T$ is a tree with root $v$. Vertices $v_{1}, \ldots, v_{d}$ are $d$ children of $v$, and $T_{i}$ is the subtree rooted by $v_{i}$. A configuration $\sigma_{\Lambda}$ is on a subset $\Lambda$ of vertices, and $R_{T}^{\sigma}$ denote the ratio of marginal probabilities at $v$ given a partial configuration $\sigma$ on $T$.

We want to study the influence of another set of vertices, say $S$, upon $v$. In particular, we want to study the range of ratios at $v$ over all possible configurations on $S$. To this end, we define the lower and upper bounds as follows. Notice that as $S$ will be fixed, we may assume that it is a subset of $\Lambda$.

Definition 23. Let $T, v, \Lambda, \sigma_{\Lambda}, S, R_{T}^{\sigma}$ be as above. Define $R_{v}:=\min _{\tau_{\Lambda}} R_{T}^{\tau_{\Lambda}}$ and $R^{v}:=$ $\max _{\tau_{\Lambda}} R_{T}^{\tau_{\Lambda}}$, where $\tau_{\lambda}$ can only differ from $\sigma_{\Lambda}$ on $S$. Define $\delta_{v}:=R^{v}-R_{v}$.

Our goal is thus to prove that $\delta_{v} \leq \exp (-\Omega(\operatorname{dist}(v, S)))$. We can recursively calculate $R_{v}$ and $R^{v}$ as follows. The base cases are:

1. $v \in S$, in which case $R_{v}=0$ and $R^{v}=\infty$ and $\delta_{v}=\infty$;

2. $v \in \Lambda \backslash S$, i.e. $v$ is fixed to be the same value in all $\tau_{\Lambda}$, in which case $R_{v}=R^{v}=0$ (or $\infty$ ) if $v$ is fixed to be blue (or green), and $\delta_{v}=0$;

3. $v \notin \Lambda$ and $v$ is the only node of $T$, in which case $R_{v}=R^{v}=\lambda$ and $\delta_{v}=0$.

For $v \notin \Lambda$, since $F_{d}$ is monotonically increasing with respect to any $x_{i}$ for any $\beta \gamma>1$,

$$
R_{v}=F_{d}\left(R_{v_{1}}, \ldots, R_{v_{d}}\right) \text { and } R^{v}=F_{d}\left(R^{v_{1}}, \ldots, R^{v_{d}}\right)
$$

where $R_{v_{i}}$ and $R^{v_{i}}$ are recursively defined lower and upper bounds of $R_{T_{i}}^{\tau_{\Lambda}}$ for $1 \leq i \leq d$. 


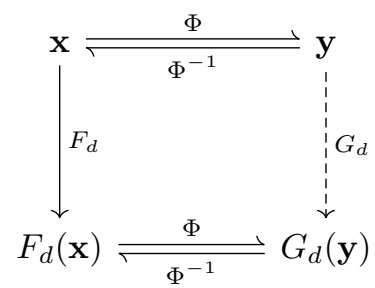

Figure 1 Commutative diagram between $F_{d}$ and $G_{d}$.

Our goal is to show that $\delta_{v}$ decays exponentially in the depth of the recursion under certain conditions such as the uniqueness. A straightforward approach would be to prove that $\delta_{v}$ contracts by a constant ratio at each recursion step. This is a sufficient, but not necessary condition for the exponential decay. Indeed there are circumstances that $\delta_{v}$ does not necessarily decay in every step but does decay in the long run. To amortize this behaviour, we use a potential function $\Phi(x)$ and show that the correlation of a new recursion decays by a constant ratio.

To be more precise, the potential function $\Phi: \mathbb{R}^{+} \rightarrow \mathbb{R}^{+}$is a differentiable and monotonically increasing function. It maps the domain of the original recursion to a new one. Let $y_{i}=\Phi\left(x_{i}\right)$. We want to consider the recursion for $y_{i}$ 's. The new recursion function, which is the pullback of $F_{d}$, is defined as

$$
G_{d}\left(y_{1}, \ldots, y_{d}\right):=\Phi\left(F_{d}\left(\Phi^{-1}\left(x_{1}\right), \ldots, \Phi^{-1}\left(x_{d}\right)\right)\right) .
$$

The relationship between $F_{d}(\mathbf{x})$ and $G_{d}(\mathbf{y})$ is illustrated in Figure 1.

We want to prove Lemma 8 and Lemma 12. To do so, we also define the upper and lower bounds of $y$. Define $y_{v}=\Phi\left(R_{v}\right)$ and accordingly $y_{v_{i}}=\Phi\left(R_{v_{i}}\right)$, for $1 \leq i \leq d$, as well as $y^{v}=\Phi\left(R^{v}\right)$ and $y^{v_{i}}=\Phi\left(R^{v_{i}}\right)$, for $1 \leq i \leq d$. We have that

$$
y_{v}=G_{d}\left(y_{v_{1}}, \ldots, y_{v_{d}}\right) \text { and } y^{v}=G_{d}\left(y^{v_{1}}, \ldots, y^{v_{d}}\right)
$$

Let $\varepsilon_{v}=y^{v}-y_{v}$. For a good potential function, exponential decay of $\varepsilon_{v}$ is sufficient to imply that of $\delta_{v}$.

- Lemma 24. Let $\Phi(x)$ be a good potential function for the field $\lambda$ at $v$. Then there exists a constant $C$ such that $\delta_{v} \leq C \varepsilon_{v}$ for any $\operatorname{dist}(v, S) \geq 2$.

Proof. By (17) and the Mean Value Theorem, there exists an $\widetilde{R} \in\left[R_{v}, R^{v}\right]$ such that

$$
\varepsilon_{v}=\Phi\left(R^{v}\right)-\Phi\left(R_{v}\right)=\Phi^{\prime}(\widetilde{R}) \cdot \delta_{v}=\varphi(\widetilde{R}) \cdot \delta_{v}
$$

Since $\operatorname{dist}(v, S) \geq 2$, we have that $R_{v} \geq \lambda \gamma^{-d}$ and $R^{v} \leq \lambda \beta^{d}$. Hence $\widetilde{R} \in\left[\lambda \gamma^{-d}, \lambda \beta^{d}\right]$, and by Condition 1 of Definition 7 , there exists a constant $C_{1}$ such that $\varphi(\widetilde{R}) \geq C_{1}$. Therefore $\delta_{v} \leq 1 / C_{1} \varepsilon_{v}$.

The next lemma explains Condition 2 of Definition 7.

- Lemma 25. Let $\Phi(x)$ be a good potential function with contraction ratio $\alpha$. Then,

$$
\varepsilon_{v} \leq \alpha \max _{1 \leq i \leq d}\left\{\varepsilon_{v_{i}}\right\}
$$


Proof. First we use (17):

$$
\varepsilon_{v}=y^{v}-y_{v}=G_{d}\left(y^{v_{1}}, \ldots, y^{v_{d}}\right)-G_{d}\left(y_{v_{1}}, \ldots, y_{v_{d}}\right) .
$$

Let $\mathbf{y}_{1}=\left(y^{v_{1}}, \ldots, y^{v_{d}}\right)$ and $\mathbf{y}_{0}=\left(y_{v_{1}}, \ldots, y_{v_{d}}\right)$. Let $\mathbf{z}(t)=t \mathbf{y}_{1}+(1-t) \mathbf{y}_{0}$ be a linear combination of $\mathbf{y}_{0}$ and $\mathbf{y}_{1}$ where $t \in[0,1]$. Then we have that

$$
\varepsilon_{v}=G_{d}(\mathbf{z}(1))-G_{d}(\mathbf{z}(0)) .
$$

By the Mean Value Theorem, there exist $\widetilde{t}$ such that $\varepsilon_{v}=\left.\frac{\mathrm{d} G_{d}(\mathbf{z}(t))}{\mathrm{d} t}\right|_{t=\widetilde{t}}$ Let $\widetilde{y_{i}}=\widetilde{t} y^{v_{i}}+(1-$ $\widetilde{t}) y_{v_{i}}$ for all $1 \leq i \leq d$. Then we have that

$$
\varepsilon_{v}=\left|\nabla G_{d}\left(\widetilde{y_{1}}, \ldots, \widetilde{y_{d}}\right) \cdot\left(\varepsilon_{v_{1}}, \ldots, \varepsilon_{v_{d}}\right)\right| .
$$

It is straightforward to calculate that

$$
\frac{\partial G_{d}(\mathbf{y})}{\partial y_{i}}=\frac{\varphi\left(F_{d}(\mathbf{R})\right)}{\varphi\left(R_{i}\right)} \cdot \frac{\partial F_{d}(\mathbf{R})}{\partial R_{i}},
$$

where $R_{i}=\Phi^{-1}\left(y_{i}\right)$ and $\mathbf{y}$ and $\mathbf{R}$ are vectors composed by $y_{i}$ 's and $R_{i}$ 's. Plugging (20) into (19) we get that

$$
\begin{aligned}
\varepsilon_{v} & =\varphi\left(F_{d}(\widetilde{\mathbf{R}})\right) \cdot \sum_{i=1}^{d}\left|\frac{\partial F_{d}}{\partial R_{i}}\right| \frac{1}{\varphi\left(\widetilde{R}_{i}\right)} \cdot \varepsilon_{v_{i}} \\
& \leq C_{\varphi, d}\left(\widetilde{R}_{1}, \ldots, \widetilde{R}_{d}\right) \cdot \max _{1 \leq i \leq d}\left\{\varepsilon_{v_{i}}\right\} \leq \alpha \max _{1 \leq i \leq d}\left\{\varepsilon_{v_{i}}\right\},
\end{aligned}
$$

where $\widetilde{R_{i}}=\Phi^{-1}\left(\widetilde{y_{i}}\right), \widetilde{\mathbf{R}}$ is the vector composed by $\widetilde{R_{i}}$ 's, and in the last line we use Condition 2 of Definition 7.

Note that the two conditions of a good potential function does not necessarily deal with all cases in the tree recursion. At the root we have one more child than other vertices in a SAW tree. Also, if $v$ has a child $u \in S$, then $\varepsilon_{u}=\infty$ and the range in both conditions of Definition 7 does not apply. To bound the recursion at the root, we have the following straightforward bound of the original recursion.

- Lemma 26. Let $(\beta, \gamma)$ be two parameters such that $\beta \gamma>1$ and $\beta<\gamma$. Let $v$ be a vertex and $v_{i}$ be its children for $1 \leq i \leq d$. Suppose $\delta_{v_{i}} \leq C$ for some $C>0$ and all $1 \leq i \leq d$. Then,

$$
\delta_{v} \leq d \lambda_{v}(\beta \gamma-1) \gamma^{-1} \beta^{d} C .
$$

Proof. It is easy to see that $\gamma \geq 1$. By the same argument as in Lemma 25 and (2), there exists $x_{i}$ 's such that

$$
\delta_{v}=\left|\nabla F_{d}\left(x_{1}, \ldots, x_{d}\right) \cdot\left(\delta_{v_{1}}, \ldots, \delta_{v_{d}}\right)\right| \leq C \sum_{i=1}^{d}\left|\frac{\partial F_{d}(\mathbf{x})}{\partial x_{i}}\right|,
$$

where $\mathbf{x}$ is the vector composed by $x_{i}$ 's. Then, we have that

$$
\left|\frac{\partial F_{d}(\mathbf{x})}{\partial x_{i}}\right|=\frac{d(\beta \gamma-1) F_{d}(\mathbf{x})}{\left(x_{i}+\gamma\right)\left(\beta x_{i}+1\right)} \leq d \lambda_{v}(\beta \gamma-1) \gamma^{-1} \beta^{d},
$$

where we use the fact that $F_{d}(\mathbf{x}) \leq \lambda_{v} \beta^{d}$ for any $x_{i} \in[0, \infty)$ and $\beta \gamma>1$. The lemma follows. 
Now we are ready to prove Lemma 8.

Proof of Lemma 8. Given $G$ and a partial configuration $\sigma_{\Lambda}$ on a subset $\Lambda \subseteq V$ of vertices, we first claim that we can approximate $p_{v}^{\sigma_{\Lambda}}$ within additive error $\varepsilon$ deterministically in time $O\left(\varepsilon^{\frac{\log \Delta}{\log \alpha}}\right)$. We construct the SAW tree $T=T_{\mathrm{SAW}}(G, v)$. Due to Proposition 3 , we only need to approximate $p_{v}^{\sigma_{\Lambda}}$ in $T$, with respect to $v$ and an arbitrary vertex set $S$. We will also use $\sigma_{\Lambda}$ to denote the configuration in $T$ on $\Lambda_{S A W}$. Let $S$ be the set of vertices whose distance to $v$ is larger than $t$, where $t$ is a parameter that we will specify later. Let $\delta_{v}$ be defined as in Definition 23 with respect to $T, v, \Lambda, \sigma_{\Lambda}$, and $S$. We want to show that $\delta_{v}=O\left(\lambda \alpha^{t}\right)$.

The maximum degree of $T$ is at most $\Delta$. Thus the root $v$ has at most $\Delta$ children in $T$, and any other vertex in $T$ has at most $\Delta-1$ children. Assume $v$ has $k \geq 1$ children as otherwise we are done. We may also assume that $v \notin S$ and let $t=\operatorname{dist}(v, S)-1 \geq 1$. We recursively construct a path $u_{0}=v, u_{1}, \ldots, u_{l}$ of length $l \leq t$ as follows. Given $u_{i}$, if there is no child of $u_{i}$, then we stop and let $l=i$. Otherwise $u_{i}$ has at least one child. If $i=t$ then we stop and let $l=t$. Otherwise $l<t$ and let $u_{i+1}$ be the child of $u_{i}$ such that $\varepsilon_{u_{i+1}}$ takes the maximum $\varepsilon$ among all children of $u_{i}$. In other words, by Lemma 25 , we have that

$$
\varepsilon_{u_{i}} \leq \alpha \varepsilon_{u_{i+1}},
$$

for all $1 \leq i \leq l-1$. Notice that (21) may not hold for $i=0$ since $v=u_{0}$ has possibly $\Delta$ children.

First we note that for all $1 \leq i \leq l, \operatorname{dist}\left(v, u_{i}\right)=i \leq l \leq t$, and therefore $u_{i} \notin S$. If we met any vertex $u_{l}$ with no child, then we claim that $\varepsilon_{u_{l}}=0$. This is because $u_{l}$ is either a free vertex with no child or $u_{l} \in \Lambda$ but $u_{l} \notin S$. However since $\varepsilon_{u_{l}}$ takes the maximum $\varepsilon$ among all children of $u_{l-1}$, we have that for all children of $u_{i-1}, \varepsilon=0$, which implies that $\varepsilon_{u_{i-1}}=0$. Recursively we get that $\varepsilon_{v}=\varepsilon_{u_{0}}=0$ and clearly the theorem holds by (18).

Hence we may assume that $l=t$. Since $u_{l} \notin S$, we have that $\delta_{u_{l}} \leq \lambda_{u_{l}} \beta^{-(\Delta-1)}$ if $\beta>1$, or $\delta_{u_{l}} \leq \lambda_{u_{l}}$ if $\beta \leq 1$. Hence by (18) and Condition 1 in Definition 7, we have that $\varepsilon_{u_{l}} \leq C_{0}$ for some constant $C_{0}$. Applying (21) inductively we have that

$$
\varepsilon_{u_{1}} \leq \alpha^{l} \varepsilon_{u_{l}} \leq \alpha^{t} C_{0} .
$$

Hence by Lemma 24, we there exists another constant $C_{1}$ such that $\delta_{u_{1}} \leq \alpha^{t} C_{1}$. To get a bound on $\delta_{u_{0}}$, we use Lemma 26, which states that

$$
\delta_{u_{0}} \leq d_{0} \lambda_{v}(\beta \gamma-1) \gamma^{-1} \beta^{d_{0}} \delta_{u_{1}} \leq d_{0} \lambda_{v}(\beta \gamma-1) \gamma^{-1} \beta^{d_{0}} \alpha^{t} C_{1}=O\left(\lambda \alpha^{t}\right),
$$

where $d_{0} \leq \Delta$ is the degree of $v=u_{0}$.

Hence the recursive procedure returns $R_{v}$ and $R^{v}$ such that $R_{v} \leq R_{T}^{\sigma_{\Lambda}} \leq R^{v}$, and $R^{v}-R_{v}=O\left(\lambda \alpha^{t}\right)$ where $\alpha<1$ is the contraction ratio. Note that $R_{T}^{\sigma_{\Lambda}}=R_{G, v}^{\sigma_{\Lambda}}=\frac{p_{v}^{\sigma_{\Lambda}}}{1-p_{v}{ }_{\Lambda}}$. Let $p_{0}=\frac{R_{v}}{R_{v}+1}$ and $p_{1}=\frac{R^{v}}{R^{v}+1}$. Then $p_{0} \leq p_{v}^{\sigma_{\Lambda}} \leq p_{1}$ and

$$
p_{1}-p_{0}=\frac{R^{v}}{R^{v}+1}-\frac{R_{v}}{R_{v}+1} \leq R^{v}-R_{v}=O\left(\lambda \alpha^{t}\right) .
$$

The recursive procedure runs in time $O\left(\Delta^{t}\right)$ since it only needs to construct the first $t$ levels of the self-avoiding walk tree. For any $\varepsilon>0$, let $t=O\left(\log _{\alpha} \varepsilon-\log _{\alpha} \lambda\right)$ so that $R^{v}-R_{v}<\varepsilon$. This gives an algorithm which approximates $p_{v}^{\sigma_{\Lambda}}$ within an additive error $\varepsilon$ in time $O\left(\left(\frac{\varepsilon}{\lambda}\right)^{\frac{\log \Delta}{\log \alpha}}\right)$.

Then we use self-reducibility to reduce computing $Z_{\beta, \gamma, \pi}(G)$ to computing conditional marginal probabilities. To be specific, let $\sigma$ be a configuration on a subset of $V$ and $\tau$ be 
sampled according to the Gibbs measure. Let $p_{v}^{\sigma}:=\operatorname{Pr}(\tau(v)=1 \mid \sigma)$ be the conditional marginal probability. We can compute $Z_{\beta, \gamma, \pi}(G)$ from $p_{v}^{\sigma}$ by the following standard procedure. Let $v_{1}, \ldots, v_{n}$ enumerate vertices in $G$. For $0 \leq i \leq n$, let $\sigma_{i}$ be the configuration fixing the first $i$ vertices $v_{1}, \ldots, v_{i}$ as follows: $\sigma_{i}\left(v_{j}\right)=\sigma_{i-1}\left(v_{j}\right)$ for $1 \leq j \leq i-1$ and $\sigma_{i}\left(v_{i}\right)$ is fixed to the spin $s$ so that $p_{i}:=\operatorname{Pr}\left(\tau\left(v_{i}\right)=s \mid \sigma_{i-1}\right) \geq 1 / 3$. This is always possible because clearly

$$
\operatorname{Pr}\left(\tau\left(v_{i}\right)=0 \mid \sigma_{i-1}\right)+\operatorname{Pr}\left(\tau\left(v_{i}\right)=1 \mid \sigma_{i-1}\right)=1 .
$$

In particular, $\sigma_{n} \in\{0,1\}^{V}$ is a configuration of $V$. The Gibbs measure of $\sigma_{n}$ is $\rho\left(\sigma_{n}\right)=$ $\frac{w\left(\sigma_{n}\right)}{Z_{\beta, \gamma, \pi}(G)}$. On the other hand, we can rewrite $\rho\left(\sigma_{n}\right)=p_{1} p_{2} \cdots p_{n}$ by conditional probabilities. Thus $Z_{\beta, \gamma, \pi}(G)=\frac{w\left(\sigma_{n}\right)}{p_{1} p_{2} \cdots p_{n}}$. The weight $w\left(\sigma_{n}\right)$ given in (1) can be computed exactly in time polynomial in $n$. Note that $p_{i}$ equals to either $p_{v_{i}}^{\sigma_{i-1}}$ or $1-p_{v_{i}}^{\sigma_{i-1}}$. Since we can approximate $p_{v}^{\sigma_{\Lambda}}$ within an additive error $\varepsilon$ in time $O\left(\left(\frac{\varepsilon}{\lambda}\right)^{\frac{\log \Delta}{\log \alpha}}\right)$, the configurations $\sigma_{i}$ can be efficiently constructed, which guarantees that all $p_{i}$ 's are bounded away from 0 . Thus the product $p_{1} p_{2} \cdots p_{n}$ can be approximated within a factor of $\left(1 \pm n \varepsilon^{\prime}\right)$ in time $O\left(n\left(\frac{\varepsilon^{\prime}}{\lambda}\right)^{\frac{\log \Delta}{\log \alpha}}\right)$. Now let $\varepsilon^{\prime}=\frac{\varepsilon}{n}$. We get the claimed FPTAS for $Z_{\beta, \gamma, \pi}(G)$.

Lemma 11 follows almost immediately from Lemmas 24, 25, and 26 as in the proof above. The only issue is that the range of $x$ should be restricted to $(0, \lambda]$. This is guaranteed by Claim 16.

Finally we show Lemma 12.

Proof of Lemma 12. By the same proof of Lemma 8, we only need to approximate the marginal probability at the root $v$ of a tree $T$. By Condition 2 of Definition $10, C_{\varphi, d}\left(x_{1}, \cdots, x_{d}\right)<$ $\alpha^{\left\lceil\log _{M}(d+1)\right\rceil}$. Denote by $B(\ell)$ the set of all vertices whose $M$-based depths of $v$ is at most $\ell$ in $T$. Hence $|B(\ell)| \leq M^{\ell}$. Let $S=\{u \mid \operatorname{dist}(u, B(\ell))>1\}$, which is essentially the same $S$ as in Lemma 8, but under a different metric. We can recursively compute upper and lower bounds $R^{v}$ and $R_{v}$ of $R_{T}^{\sigma_{\Lambda}}$ such that $R_{v} \leq R_{T}^{\sigma_{\Lambda}} \leq R^{v}$, with the base case that for any vertex $u \in S$ trivial bounds $R_{u}=0$ and $R^{u}=\infty$ are used.

We proceed as in the proof of Lemma 8 . Without loss of generality, we construct a path $u_{0} u_{1} \cdots u_{k}$ in $T$ from the root $u_{0}=v$ to a $u_{k}$ with $\ell_{M}\left(u_{k-1}\right) \leq \ell$ and $\ell_{M}\left(u_{k}\right)>\ell$. As in the proof of Lemma 25, $\varepsilon_{u_{j}} \leq C_{d_{j}}^{\varphi}\left(x_{j, 1}, \ldots, x_{j, d_{j}}\right) \cdot \varepsilon_{u_{j+1}}$ for all $0 \leq j \leq k-1$, where $d_{j}$ is the number of children of $u_{j}$ and $x_{j, i} \in[0, \infty), 1 \leq i \leq d_{j}$. Hence we have that

$$
\begin{aligned}
\varepsilon_{v} & \leq \varepsilon_{u_{k}} \cdot \prod_{j=0}^{k-1} \alpha^{\left\lceil\log _{M}\left(d_{j}+1\right)\right\rceil} \leq \varepsilon_{u_{k}} \cdot \alpha^{\sum_{j=0}^{k-1}\left\lceil\log _{M}\left(d_{j}+1\right)\right\rceil} \\
& =\varepsilon_{u_{k}} \cdot \alpha^{\ell_{M}\left(u_{k}\right)} \leq \varepsilon_{u_{k}} \cdot \alpha^{\ell} .
\end{aligned}
$$

Note that $\operatorname{dist}\left(u_{k}, B(\ell)\right)=1$ and hence $u_{k} \notin S$. So $\delta_{u_{k}}<\lambda_{u_{k}} \leq \lambda$. By (18), we have that $\varepsilon_{u_{k}} \leq \varphi(\widetilde{R}) \delta_{u_{k}}$, for some $\widetilde{R} \in\left[\lambda_{u_{k}} \gamma^{-d_{k}}, \lambda_{u_{k}} \beta^{d_{k}}\right]$. Hence $\varepsilon_{u_{k}}<C_{2} \lambda$ by Condition 1 of Definition 10, and $\varepsilon_{v}<\lambda \alpha^{\ell} C_{2}$. By (18) and Condition 1 of Definition 10 again, we have that $\delta_{v} \leq \lambda \alpha^{\ell} C_{2} / C_{1}$.

The rest of the proof goes the same as that of Lemma 8. The running time has an extra $n^{2}$ factor since we need to go down two more levels (in the worst case) outside of $B(\ell)$.

\subsection{Proofs of Lemma 14 and Lemma 15}

In this section we show Lemma 14 and Lemma 15. We prove Lemma 14 first, and then use it to show Lemma 15. 
Proof of Lemma 14. It is trivial if $\beta \leq 1$. Now assume that $\beta>1$. As $\frac{\beta x+1}{x+\gamma}$ is increasing in $x$, it is equivalent to show that

$$
\frac{\gamma-1}{\beta-1} \geq \lambda_{c}=\left(\frac{\gamma}{\beta}\right)^{\frac{\sqrt{\beta \gamma}}{\sqrt{\beta \gamma}-1}} \quad \Leftrightarrow \quad \log (\gamma-1)-\log (\beta-1) \geq \frac{\sqrt{\beta \gamma}}{\sqrt{\beta \gamma}-1} \log \left(\frac{\gamma}{\beta}\right) .
$$

Let $\gamma=k^{2} \beta$ with $k \geq 1$. We only need to show that $r(k) \geq 0$ for $k \geq 1$, where $r(k)$ is defined as

$$
r(k):=\log \left(\beta k^{2}-1\right)-\log (\beta-1)-\frac{2 \beta k}{\beta k-1} \log k .
$$

Since $r(1)=0$, it is enough to prove that $r(k)$ is increasing for $k \geq 1$. It can be easily verified as

$$
\begin{aligned}
r^{\prime}(k) & =\frac{2 \beta k}{\beta k^{2}-1}-\frac{2 \beta}{\beta k-1}+\frac{2 \beta}{(\beta k-1)^{2}} \log k \\
& =\frac{2 \beta}{(\beta k-1)^{2}\left(\beta k^{2}-1\right)}\left(\left(\beta k^{2}-1\right) \log k-(k-1)(\beta k-1)\right) .
\end{aligned}
$$

So, it is sufficient to show that

$$
\left(\beta k^{2}-1\right) \log k-(k-1)(\beta k-1) \geq 0 .
$$

Since $k \geq 1$, we have that $\log k \geq 1-\frac{1}{k}$. It implies that

$$
\left(\beta k^{2}-1\right) \log k-(k-1)(\beta k-1) \geq\left(\beta k^{2}-1\right)\left(1-\frac{1}{k}\right)-(k-1)(\beta k-1)=\frac{(k-1)^{2}}{k} \geq 0 .
$$

This completes the proof.

Then we show Lemma 15.

Proof of Lemma 15. Let $g(x):=(\beta \gamma-1) x \log \frac{\lambda_{c}}{x}-(\beta x+1)(x+\gamma) \log \frac{x+\gamma}{\beta x+1}$. Hence it is equivalent to show that $g(x) \leq 0$ for all $0<x<\lambda_{c}$. Take the derivative of $g(x)$ and we have that

$$
\begin{aligned}
g^{\prime}(x)= & (\beta \gamma-1)\left(\log \frac{\lambda_{c}}{x}-1\right)-(2 \beta x+\beta \gamma+1) \log \frac{x+\gamma}{\beta x+1} \\
& -(\beta x+1)(x+\gamma)\left(\frac{1}{x+\gamma}-\frac{\beta}{\beta x+1}\right) \\
= & (\beta \gamma-1) \log \frac{\lambda_{c}}{x}-(2 \beta x+\beta \gamma+1) \log \frac{x+\gamma}{\beta x+1} .
\end{aligned}
$$

By direct calculation, $g\left(\sqrt{\frac{\gamma}{\beta}}\right)=0$ and $g^{\prime}\left(\sqrt{\frac{\gamma}{\beta}}\right)=0$. Then we prove (3) for the case of $0<x<\sqrt{\frac{\gamma}{\beta}}$ and $\sqrt{\frac{\gamma}{\beta}}<x<\lambda_{c}$ separately.

If $0<x<\sqrt{\frac{\gamma}{\beta}}$, it is sufficient to verify that $g^{\prime}(x)>0$. We only need to show that $g^{\prime}(x)$ is decreasing since $g^{\prime}\left(\sqrt{\frac{\gamma}{\beta}}\right)=0$. It is easily verified by taking the derivative again:

$$
\begin{aligned}
g^{\prime \prime}(x) & =-\frac{\beta \gamma-1}{x}-2 \beta \log \frac{x+\gamma}{\beta x+1}-(2 \beta x+\beta \gamma+1)\left(\frac{1}{x+\gamma}-\frac{\beta}{\beta x+1}\right) \\
& =-2 \beta \log \frac{x+\gamma}{\beta x+1}-(\beta \gamma-1)\left(\frac{1}{x}-\frac{2 \beta x+\beta \gamma+1}{(x+\gamma)(\beta x+1)}\right) \\
& =-2 \beta \log \frac{x+\gamma}{\beta x+1}-(\beta \gamma-1) \frac{r-\beta x^{2}}{x(x+\gamma)(\beta x+1)}<0,
\end{aligned}
$$


where the last inequality uses the fact that $\frac{x+\gamma}{\beta x+1} \geq 1$ by Lemma 14 and $x<\sqrt{\frac{\gamma}{\beta}}$.

If $\sqrt{\frac{\gamma}{\beta}}<x<\lambda_{c}$, then we show (3) directly. First notice that as $x \neq \sqrt{\frac{\gamma}{\beta}}$,

$$
\frac{x}{(\beta x+1)(x+\gamma)}=\frac{1}{\beta x+\frac{\gamma}{x}+\beta \gamma+1}<(\sqrt{\beta \gamma}+1)^{-2},
$$

Given this, in order to get (3), it is sufficient to show that $h(x)<0$ where

$$
h(x):=\frac{\sqrt{\beta \gamma}-1}{\sqrt{\beta \gamma}+1} \log \frac{\lambda_{c}}{x}-\log \frac{x+\gamma}{\beta x+1} .
$$

In fact, $h(x)$ is a decreasing function as

$$
\begin{aligned}
h^{\prime}(x) & =-\frac{\sqrt{\beta \gamma}-1}{x(\sqrt{\beta \gamma}+1)}-\frac{1}{x+\gamma}+\frac{\beta}{\beta x+1} \\
& =-\frac{(\sqrt{\beta \gamma}-1)\left((x+\gamma)(\beta x+1)-(\sqrt{\beta \gamma}+1)^{2} x\right)}{x(\sqrt{\beta \gamma}+1)(x+\gamma)(\beta x+1)} \\
& =-\frac{(\sqrt{\beta \gamma}-1)(\sqrt{\beta} x-\sqrt{\gamma})^{2}}{x(\sqrt{\beta \gamma}+1)(x+\gamma)(\beta x+1)} \leq 0 .
\end{aligned}
$$

Notice that $h\left(\sqrt{\frac{\gamma}{\beta}}\right)=0$. It implies that $h(x)<0$ for all $x>\sqrt{\frac{\gamma}{\beta}}$. This completes the proof.

Acknowledgement. We thank Liang Li, Jingcheng Liu, and Chihao Zhang for some stimulating discussion. In particular, the example of the 5-7 tree in Section 5 is an outcome from such discussion. We also thank organizers of the "IMA-GaTech Workshop on the Power of Randomness in Computation" in March 2015. The current work stems from discussions during the workshop.

\section{References}

1 Jin-Yi Cai, Andreas Galanis, Leslie Ann Goldberg, Heng Guo, Mark Jerrum, Daniel Štefankovič, and Eric Vigoda. \#BIS-hardness for 2-spin systems on bipartite bounded degree graphs in the tree non-uniqueness region. In RANDOM, pages 582-595, 2014.

2 Jin-Yi Cai and Michael Kowalczyk. Spin systems on $k$-regular graphs with complex edge functions. Theor. Comput. Sci., 461:2-16, 2012.

3 Martin E. Dyer, Leslie Ann Goldberg, Catherine S. Greenhill, and Mark Jerrum. The relative complexity of approximate counting problems. Algorithmica, 38(3):471-500, 2003.

4 Andreas Galanis, Daniel Štefankovič, and Eric Vigoda. Inapproximability of the partition function for the antiferromagnetic Ising and Hard-Core models. CoRR, 2012. URL: http: //arxiv.org/abs/1203.2226.

5 Hans-Otto Georgii. Gibbs Measures and Phase Transitions, volume 9 of De Gruyter Studies in Mathematics. de Gruyter, Berlin, second edition, 2011.

6 Leslie Ann Goldberg and Mark Jerrum. The complexity of ferromagnetic Ising with local fields. Combinatorics, Probability \& Computing, 16(1):43-61, 2007.

7 Leslie Ann Goldberg, Mark Jerrum, and Mike Paterson. The computational complexity of two-state spin systems. Random Struct. Algorithms, 23(2):133-154, 2003.

8 Mark Jerrum and Alistair Sinclair. Polynomial-time approximation algorithms for the Ising model. SIAM J. Comput., 22(5):1087-1116, 1993. 
9 Mark Jerrum, Leslie G. Valiant, and Vijay V. Vazirani. Random generation of combinatorial structures from a uniform distribution. Theor. Comput. Sci., 43:169-188, 1986.

10 Frank P. Kelly. Stochastic models of computer communication systems. Journal of the Royal Statistical Society. Series B (Methodological), 47(3):379-395, 1985.

11 Liang Li, Pinyan Lu, and Yitong Yin. Approximate counting via correlation decay in spin systems. In SODA, pages 922-940, 2012.

12 Liang Li, Pinyan Lu, and Yitong Yin. Correlation decay up to uniqueness in spin systems. In $S O D A$, pages $67-84,2013$.

13 Jingcheng Liu, Pinyan Lu, and Chihao Zhang. The complexity of ferromagnetic two-spin systems with external fields. In RANDOM, pages 843-856, 2014.

14 Russell Lyons. The Ising model and percolation on trees and tree-like graphs. Comm. Math. Phys., 125(2):337-353, 1989.

15 Elchanan Mossel and Allan Sly. Exact thresholds for Ising-Gibbs samplers on general graphs. Annals of Probability, 41(1):294-328, 2013.

16 Alistair Sinclair, Piyush Srivastava, Daniel Štefankovič, and Yitong Yin. Spatial mixing and the connective constant: Optimal bounds. In SODA, pages 1549-1563, 2015.

17 Alistair Sinclair, Piyush Srivastava, and Marc Thurley. Approximation algorithms for twostate anti-ferromagnetic spin systems on bounded degree graphs. In SODA, pages 941-953, 2012 .

18 Allan Sly and Nike Sun. The computational hardness of counting in two-spin models on d-regular graphs. The Annals of Probability, 42(6):2383-2416, 2014.

19 Dror Weitz. Counting independent sets up to the tree threshold. In STOC, pages 140-149, 2006.

20 Jinshan Zhang, Heng Liang, and Fengshan Bai. Approximating partition functions of the two-state spin system. Inf. Process. Lett., 111(14):702-710, 2011. 